\title{
Epigenetic Modulators
}

\author{
Kenneth W. Duncan and John E. Campbell
}

\begin{abstract}
Epigenetic drug discovery has expanded significantly beyond histone deactylases (HDAC) in the past decade. This review covers significant new advances in the field across three more targets classes: bromodomains (BRD), arginine/lysine methyltransferases (RMT/KMT), and lysine demethylases (KDM). In each target section, a broad overview from a medicinal chemistry perspective is presented covering high-quality chemical biology tools being used to further expand the innate role of each target in cancer biology and in some examples facilitating preclinical target validation. In each target space, the review will also cover candidates currently in clinical investigation.
\end{abstract}

Keywords Bromodomain, Cancer, Demethylase, Epigenetics, Methyltransferase

\section{Contents}

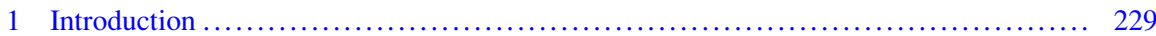

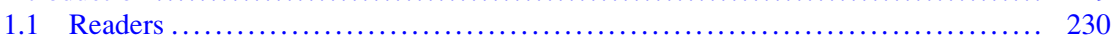

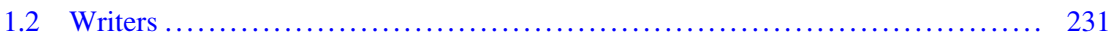

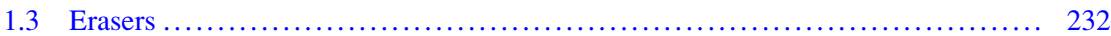

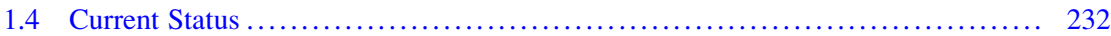

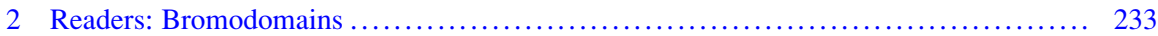

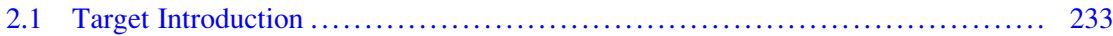

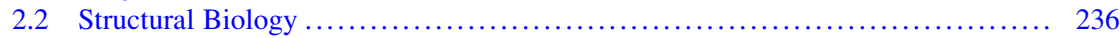

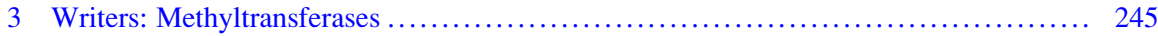

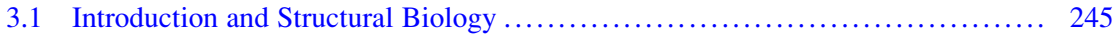

3.2 Arginine Methyltransferases ........................................ 245

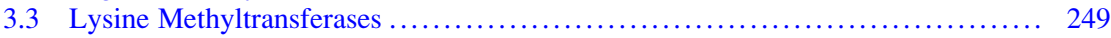

4 Erasers: Lysine Demethylases ......................................... 266

K.W. Duncan ( $\triangle$ ) and J.E. Campbell

Molecular Discovery, Epizyme, Inc., Cambridge, MA, USA

e-mail: kduncan@epizyme.com 


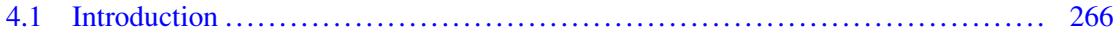

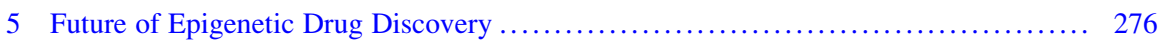

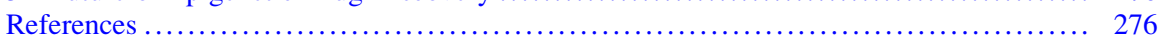

\section{Abbreviations}

Ac

Acetyl

ALL Acute lymphoblastic leukemia

ApoA1 Apolipoprotein A1

ASN

Aspartame

ATAD2 ATPase family AAA domain-containing protein 2

ATP

Adenosine triphosphate

BAP1

BRCA1-associated protein-1

BAZ

Bromodomain adjacent to zinc finger domain protein

BET

Bromodomain and extra-terminal motif

BRD Bromodomain

BRET Bioluminescence resonance energy transfer

BTK Bruton's tyrosine kinase

CHD Chromodomain

CREBBP CREB-binding protein

DNA Deoxyribonucleic acid

DNMT DNA methyltransferase

DOT1L Disruptor of telomeric silencing 1-like

E2F E2 factor

EBV Epstein-Barr virus

EHMT Euchromatin histone methyltransferase

EZH2 Enhancer of zeste 2 polycomb repressive complex 2 subunit

HAT Histone acetyltransferase

HDAC Histone deacetylase

HMT Histone methyltransferase

JAK Janus kinase

JMJD JmjC domain-containing protein

KAT Lysine acetyltransferase

KDM Lysine demethylase

KMT Lysine methyltransferase

LOF Loss of function

LSD1 Lysine-specific histone demethylase 1

MBT Malignant brain tumor

MEKK MAP kinases kinase kinase

MEP50 Methylsome

MLL Mixed lineage leukemia

mRNA Messenger ribonucleic acid

NHL Non-Hodgkin lymphoma

NUT Nuclear protein of testis 


$\begin{array}{ll}\text { 2OG } & \text { 2-Oxoglutarate } \\ \text { PARP1 } & \text { Poly(ADP-ribose) polymerase 1 } \\ \text { PDB } & \text { Protein Data Bank } \\ \text { PHD } & \text { Plant homeodomain } \\ \text { PMT } & \text { Protein methyltransferase } \\ \text { PRC2 } & \text { Polycomb repressive complex } \\ \text { PRMT } & \text { Protein arginine methyltransferase } \\ \text { RMT } & \text { Arginine methyltransferase } \\ \text { RNA pol } & \text { RNA polymerase } \\ \text { SAH } & \text { S-Adenosyl-L-homocysteine } \\ \text { SAM } & \text { S-Adenosyl methionine } \\ \text { SCLC } & \text { Small cell lung cancer } \\ \text { SET } & \text { Su(var)3-9 and "enhancer of zeste" proteins } \\ \text { SETDB1 } & \text { SET domain bifurcated 1 } \\ \text { SFG } & \text { Sinefungin } \\ \text { SGC } & \text { Structural genomics consortium } \\ \text { SMARCA } & \text { SWI/SNF-related matrix-associated actin-dependent regulator chromatin } \\ \text { SMYD } & \text { SET and MYND domain-containing protein } \\ \text { STAT } & \text { Signal transducer and activator of transcription } \\ \text { SUV39H1 } & \text { Suppressor of variegation 3-9 homolog 1 } \\ \text { SWI/SNF } & \text { SWItch/sucrose non-fermentable } \\ \text { TCGA } & \text { The Cancer Genome Atlas } \\ \text { TGFb } & \text { Transforming growth factor beta } \\ \text { TLR } & \text { Toll-like receptor } \\ \text { TRIM24 } & \text { Tripartite motif-containing 24 } \\ \text { TWIST } & \text { Twist-related protein } \\ \text { WHO } & \text { World Health Organization } \\ & \end{array}$

\section{Introduction}

To accommodate approximately $2 \mathrm{M}$ of DNA into the nucleus of each cell, DNA is coiled around a core of histone proteins with the resulting tightly packaged chromatin units creating the well-known structure of the chromosome. The fully condensed chromatin state, known as heterochromatin, sterically limits access of gene promoter regions to transcription factors, polymerases, and other components for gene transcription. A less compacted state, known as euchromatin, is required before transcriptional machinery can access promoter genes and commence transcription (Fig. 1).

Epigenetic mechanisms are the processes that modulate chromatin structure, gating access to gene promoter regions for transcriptional machinery whether activating or repressive. These physicochemical mechanisms driving chromatin remodeling can be described under one of three broad categories, namely, (Type A) covalent modification of chromosomal DNA, (Type B) posttranslational 

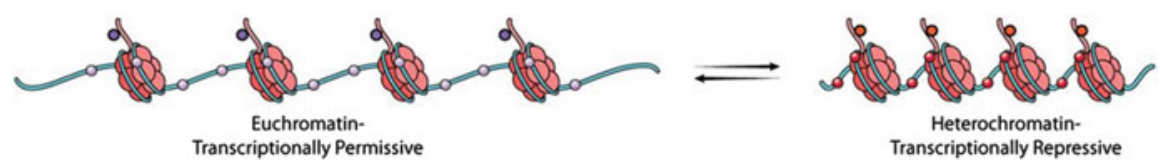

Fig. 1 Transcriptional activities of euchromatin and heterochromatin

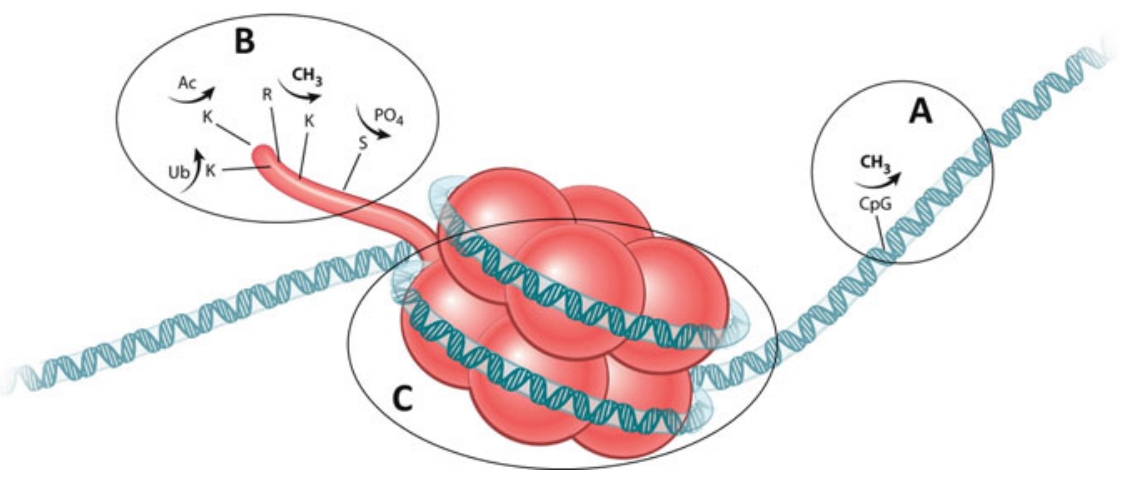

Fig. 2 Posttranslational epigenetic modifications of chromatin histone proteins and DNA

covalent modifications to histone tails, and (Type C) ATP hydrolysis-mediated alteration of DNA-histone interactions (Fig. 2).

A plethora of proteins interact with epigenetic "marks" (i.e., covalent modifications) to chromosomal DNA, histones, or other protein targets. These proteins contain domains capable of performing protein recognition based on these sitespecific markings (readers) or are responsible for the addition (writers) or removal (erasers) of these marks (Fig. 3).

\subsection{Readers}

Histone acetyl and methyl marks are read by proteins containing one or more specialized reader domains with most of the methyl-reading domains belonging to the highly conserved "Royal" superfamily of homologs originally identified in plant or Drosophila chromatin-remodeling proteins [1]. The regulatory reader domain family members with relevant homologs in man include Tudor, malignant brain tumor (MBT), chromodomain (CHD), bromodomain (BRD), and plant homeodomain (PHD), among others. Tudor domain-containing proteins interact with di- and trimethylated lysine and are also known to be able to interact with methylated arginine. The MBT domain-containing proteins specifically bind monoand dimethylated lysines. Methylation marks localized to the $\mathrm{H} 3$ histone subunit tail region can also be read by chromodomains, whereas bromodomains recognize only N-Ac residues on these same histone tails. PHD domains, in contrast, can recognize lysine acetylation and all three methylation states. At the time of writing this chapter, only one member of the methyl-reading MBT domain (L3MBTL3) has 


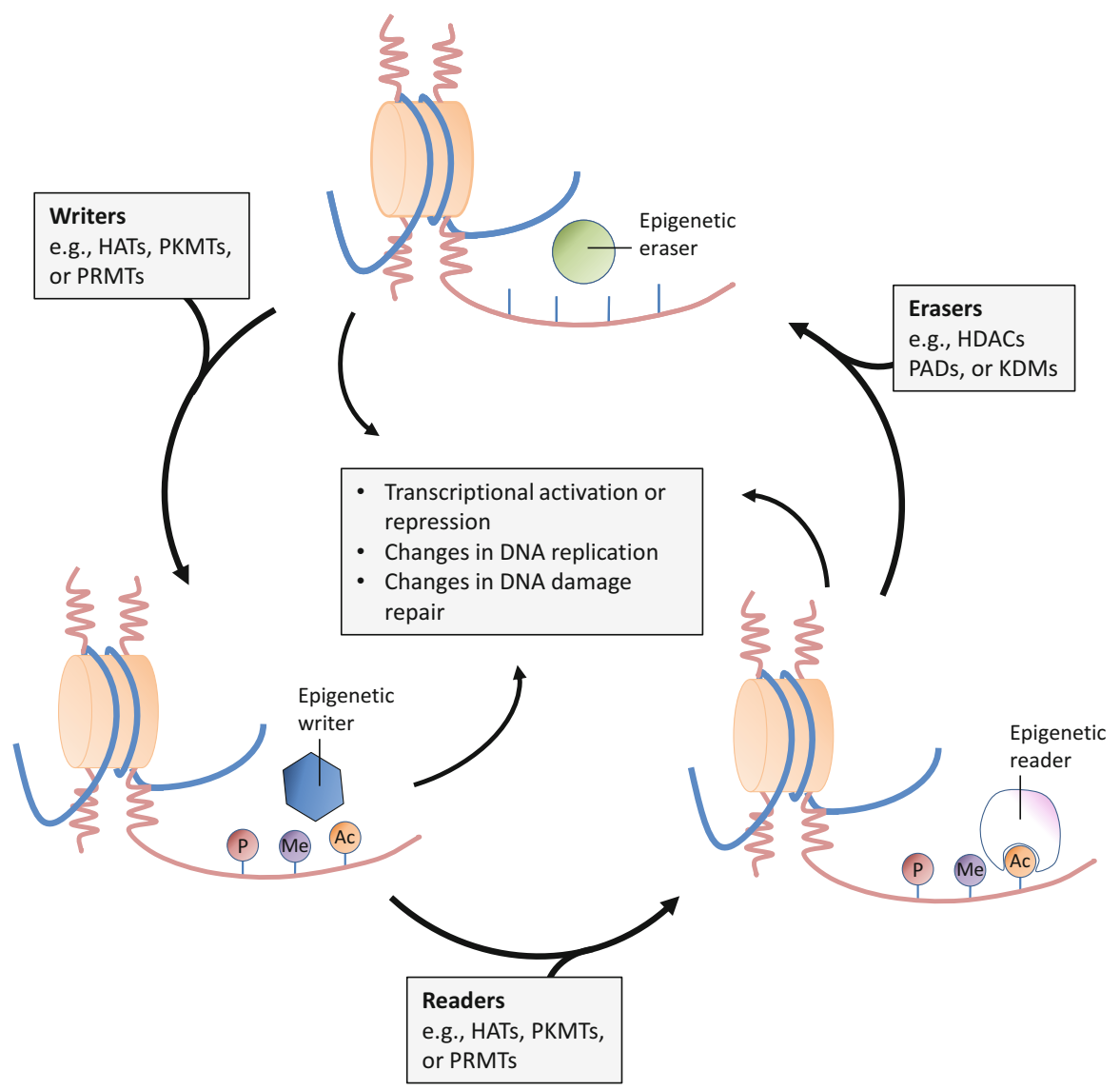

Fig. 3 The three different types of epigenetic modifiers that are responsible for installing, removing, and recognizing posttranslational modifications are known as writers, erasers, and readers

a validated inhibitor [2-4], while several bromodomain inhibitors have transitioned into clinical trials. Accordingly, this chapter will highlight the significant progress in development in targeting acetyl reader domains with a focus on BRD inhibitors.

\subsection{Writers}

Covalent modification of histone residues, commonly via methylation and acetylation, is a dynamic process that is utilized as a kind of code that, once recognized by reader domains, is translated into a relaxing or tightening of the chromatin complex to regulate transcription. Addition of up to two methyl groups to the $\omega-N$ atoms of arginine residues can be performed by a family of enzymes known as the arginine methyltransferases (RMTs). Similarly, lysine residues on histones can undergo $\varepsilon-N$ methylation up to three times by lysine methyltransferases (KMTs). Lysine residues 
can also undergo covalent modification by one of the 18-member family of histone acetyltransferase (HAT or KATs) enzymes which mediate the mono-transfer of an acetate moiety to lysine residues from acetyl-CoA on histones and other proteins. At the time of writing this chapter, the HAT enzyme family has not been studied extensively; however, some putative inhibitors have been described in the literature [5-7]. Again, reflecting significant advances in the field over the past 10 years, our focus in this chapter will be on the protein methyltransferases for which clinical candidates have now been realized.

\subsection{Erasers}

Histone deacetylases (HDACs) were the first class of epigenetic erasers to be discovered and targeted for the treatment of human disease including cancer. As presented in the first volume of this title, HDAC enzymes are responsible for the removal of acetyl marks, and the blockade of this mechanism has led to the successful creation of pan-HDAC inhibitor drugs and more, recently, selective HDAC inhibitors that are currently in clinical trials.

Despite the discovery of deacetylase enzymes, lysine methylation was initially thought to be a static process, until the Zhang group demonstrated, for the first time, that lysine methylation was indeed reversible [8,9]. Since the initial demethylase report, however, eight classes of lysine demethylases (KDMs) have now been reported even though their biological roles are still being defined. Interestingly however, despite a number of conflicting reports around the nonheme iron(II) and 2-oxoglutarate (2OG)-dependent oxygenase JMJD6 [10, 11], there appears to be no known enzyme capable of demethylation of modified arginine residues. However, arginine residues can be converted to citrulline by the arginine deiminase family of enzymes [12]. This chapter will provide a summary of the development of KDM inhibitors currently in early discovery and early stage clinical trials.

\subsection{Current Status}

Epigenetic drug discovery has changed much in the past 10 years. Since the first approval of azacitidine in 2004, drugs targeting epigenetic mechanisms have altered the clinical landscape for oncology patients. Hypomethylating agents, such as azacitidine and in 2006 decitabine, both act through limiting DNA methyltransferase (DNMT) activity as pseudo-substrates and are generally viewed as examples of the Type A chromatin modulators described above (Fig. 1). 
The first example of a drug targeting a Type B epigenetic target, vorinostat, was approved in late 2006 acting as a pan-active histone deacetylase (HDAC) inhibitor [13]. A number of promising HDAC inhibitors were discussed in volume 1 of this title [14] and will not be discussed further in this chapter. Although a number of additional FDA approvals have followed for pan-active HDAC inhibitors (Table 1), no enzyme selective or subclass selective HDAC inhibitors (e.g., type I, type IIa, etc.) have successfully completed clinical trials to date. Drug discovery efforts have continued from a number of groups, leading to high-quality, class-specific inhibitors to facilitate further understanding of the underlying biology of these HDAC targets, developing new clinical hypotheses in oncology and beyond. This drug discovery effort has continued across a spectrum of epigenetic targets with a number currently being investigated in the clinic (Table 1).

In this chapter, we will cover the most recent epigenetic targets to progress into evaluation in a clinical setting, focusing on the rapid progress made on targets that utilize methyl- and acetyl-based epigenetic marks on histones and other proteins. This chapter will also review some additional epigenetic targets which have highquality tool compounds available to test new therapeutic hypotheses and may provide a future path for novel, targeted therapies in oncology.

\section{Readers: Bromodomains}

\subsection{Target Introduction}

First described in the 1990s as part of the brahma gene from Drosophila melanogaster [15], the bromodomains are a set of evolutionarily conserved protein interaction modules capable of binding to, and thus recognizing, $\varepsilon-N$-acetylation of lysine residues. A total of 61 bromodomains are encoded within the human proteome and can be found in a diverse set of 46 nuclear and cytoplasmic proteins [16]. These proteins can contain one or more acetyl readers in addition to other domains which may contribute to a range of chromatin functions, including writing of acetyl $[17,18]$ or methyl groups [19, 20], demethylation [21], or larger chromatin remodeling roles as part of a larger complex [22, 23]. Further phylogenic analysis of these 61 domains has led to the definition of eight structural classes (Fig. 4).

A number of bromodomains have been linked to cancer indications [24] through aberrant expression levels, mutations, or genetic rearrangements of bromodomaincontaining proteins, either in the form of wild-type domain rearrangements or translocations. The most studied of these in cancer drug discovery can be found on branch II of the phylogenic tree containing the bromodomain and extra C-terminal (BET) subfamily consisting of multiple isoforms of BRD2, BRD3, BRD4, and BRDT. BET proteins contain conserved tandem $\mathrm{N}$-terminal bromodomains (BD1 and BD2), which allow for interaction with acetylated histones and various nuclear proteins. As functional readers of acetylated chromatin, these proteins mediate 
Table 1 Clinical status of epigenetic focused therapies targeting enzymes which modulate acetylation or methylation status

\begin{tabular}{|c|c|c|c|}
\hline Drug name & Target class/enzyme & Status & Indication \\
\hline Belinostat & HDAC/pan-HDAC & Approved & Peripheral T-cell lymphoma \\
\hline Panobinostat & HDAC/pan-HDAC & Approved & Multiple myeloma \\
\hline Romidepsin & $\begin{array}{l}\text { HDAC/Zn-dependent } \\
\text { HDACS }\end{array}$ & Approved & Cutaneous T-cell lymphoma \\
\hline Vorinostat & HDAC/pan-HDAC & Approved & Cutaneous T-cell lymphoma \\
\hline Pracinostat & HDAC/class I, II, IV & Approved & AML, T-cell lymphoma \\
\hline Entinostat & HDAC/class I, III & $\begin{array}{l}\text { Phase } \\
\text { II/III }\end{array}$ & Multiple cancers \\
\hline Givinostat & HDAC/class I, II & Phase II & \\
\hline Mocetinostat & HDAC/ pan-HDAC & Phase II & Lymphoma, leukemia \\
\hline Quisinostat & HDAC/pan-HDAC & Phase II & $\begin{array}{l}\text { Leukemia, MLL, lymphoma, solid } \\
\text { malignancies }\end{array}$ \\
\hline Resminostat & HDAC & Phase II & $\mathrm{HCC}$ \\
\hline Tacedinaline & $\begin{array}{l}\text { HDAC/HDAC1, } \\
\text { HDAC2 }\end{array}$ & Phase II & Multiple myeloma \\
\hline Valproic acid & HDAC/pan-HDAC & Phase II & Multiple cancers \\
\hline ACY-1215 & HDAC/HDAC6 & Phase I/II & Multiple myeloma \\
\hline AR-42 & HDAC/pan-HDAC & Phase I/II & MLL, lymphoma, leukemia \\
\hline CUDC-907 & HDAC/class I, IIB & Phase I & $\begin{array}{l}\text { Lymphoma, multiple myeloma, solid } \\
\text { tumors }\end{array}$ \\
\hline Abexinostat & HDAC/pan-HDAC & Phase I & B-cell lymphoma \\
\hline $\begin{array}{l}\text { MK-8628/ } \\
\text { OTX015 }\end{array}$ & Bromodomain/BET & Phase I/II & Glioblastoma \\
\hline INCB054329 & Bromodomain & Phase I/II & Advanced malignancies \\
\hline ABBV-075 & Bromodomain & Phase I & Advanced cancers \\
\hline BAY-1238097 & Bromodomain/BET & Phase I & Neoplasms \\
\hline BMS-986158 & Bromodomain/BET & Phase I & Solid tumors \\
\hline CPI-0610 & Bromodomain/BET & Phase I & Lymphoma \\
\hline TEN-010 & Bromodomain/BET & Phase I & AML and solid tumors \\
\hline Tazemetostat & HMT/EZH2 & Phase II & $\begin{array}{l}\text { Lymphoma, INI-deficient tumors, } \\
\text { synovial sarcoma }\end{array}$ \\
\hline CPI-1205 & HMT/EZH2 & Phase I & B-cell lymphoma \\
\hline GSK2816126 & HMT/EZH2 & Phase I & $\begin{array}{l}\text { B-cell lymphoma, follicular } \\
\text { lymphoma }\end{array}$ \\
\hline Pinometostat & HMT/DOT1L & Phase I & Leukemia \\
\hline ORY-1001 & KDM/KDM1A & Phase I/II & AML \\
\hline Tranylcypromine & KDM/KDM1A & Phase I/II & AML \\
\hline GSK2879552 & KDM/KDM1A & Phase I & AML \\
\hline
\end{tabular}

interactions of $\mathrm{BD} 1$ and $\mathrm{BD} 2$, allowing for discrete localization of BET proteins at various acetylated chromosomal locations, thus permitting these BET proteins to recruit regulatory complexes and influence gene expression. 


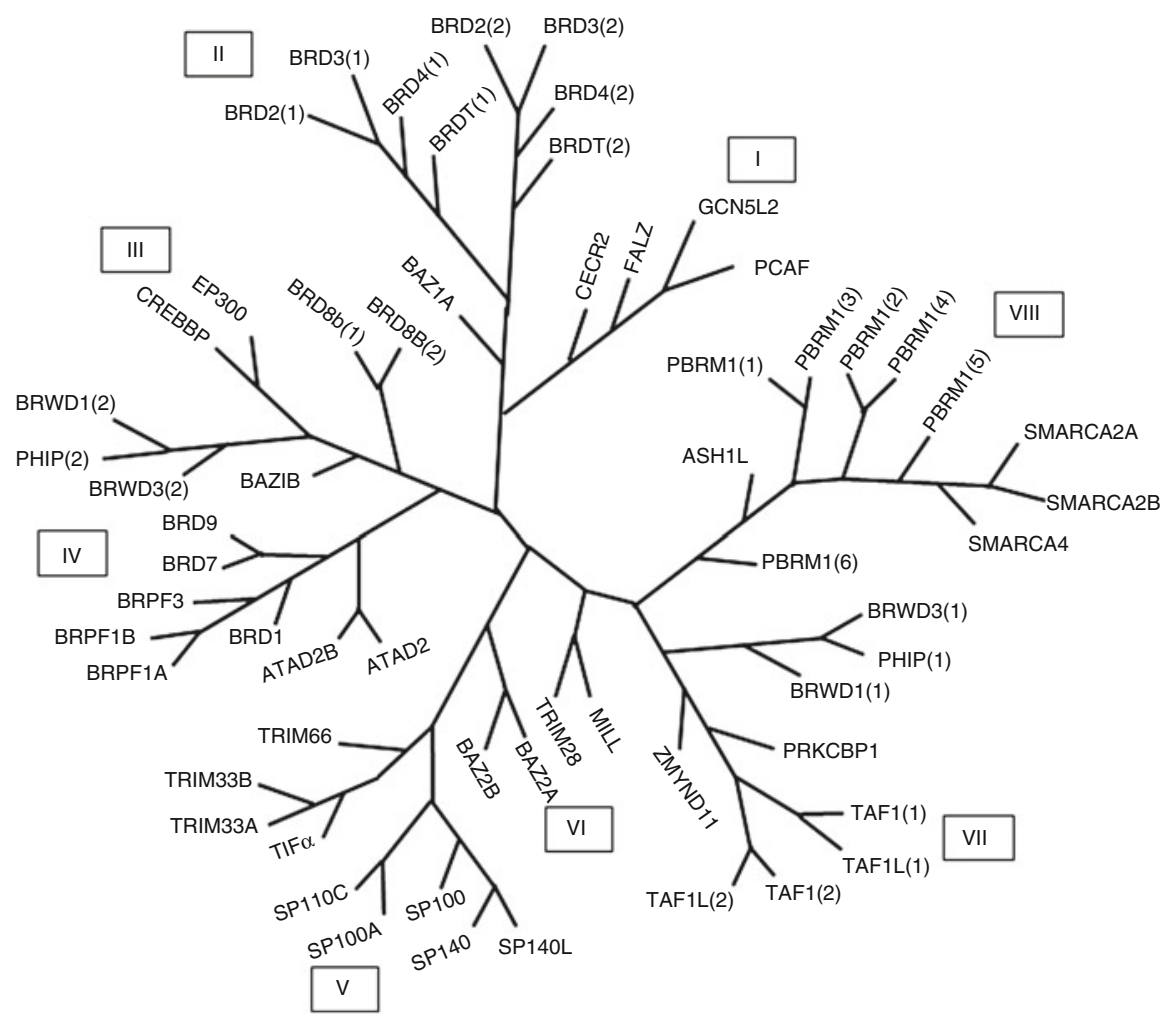

Fig. 4 Phylogenetic tree containing bromodomains

CREBBP (CREB-binding protein) with related protein EP300 have both acetyltransferase (HAT) and bromodomain (BET) regions. CREBBP/EP300-mediated acetylation creates binding sites for the acetyl-lysine-specific CREBBP/EP300 bromodomain, which has been postulated to be required for accurate substrate targeting, the results of which is a positive feedback loop and corresponding maintenance of CREBBP/EP300 enzymatic activity [25]. Chromosomal translocations of CREBBP/EP300 have been described in myeloid and acute lymphoid leukemia and may also contribute to tumorigenesis of NUP-HoxA9 and MOZ-TIF2 fusion proteins by activation of transcription [26, 27]. It is currently unclear which function is ideal as an anticancer target; however, inhibitors of both the HAT and BRD domains have been published recently [6, 28, 29].

Overexpression of bromodomain ATAD2 (ATPase family AAA domaincontaining protein 2) occurs in some $70 \%$ of breast tumors and correlates with poor survival and a propensity for relapse [30] as is required recruitment of specific 
E2F transcription factors and for chromatin assembly of the host cell factor 1-MLL histone methyltransferase complex. Mutations in ATAD2 can effectively disable this to promote cancer cell proliferation [31]. Closely related protein ATAD2B has recently been shown to be highly expressed in glioblastoma and oligodendroglioma, as well as in breast carcinoma [32].

Tripartite motif 24 protein (TRIM24) overexpression has been associated with poor overall survival of breast cancer patients [33], and high expression is reported in samples of numerous other cancer types such as hepatocellular carcinoma, glioblastoma, gastric cancer, non-small cell lung cancer, and head and neck squamous cancer [34-38]. Through additional mechanisms still being unearthed, TRIM24 may regulate levels of cancer-relevant proteins on the transcriptional and protein levels [39].

This section will however focus upon the BET bromodomains implicated in cancer highlighting the compounds currently in early clinical assessment. We will also review some examples of the breadth and depth of inhibitor chemotypes identified across the bromodomain family, with which additional chemical biology studies may enable validation in a wider range of cancer indications. An exhaustive review of bromodomain inhibitor chemical matter was written by Filippakopoulos and Knapp in 2014 [16].

\subsection{Structural Biology}

Crystallographic data have been collected on a number of bromodomains and first described in detail by Mutjaba et al. [40]. Although a large degree of sequence variation exists in the bromodomain modules, they shared a conserved tertiary structure. This feature consists of a left-handed bundle of four $\alpha$-helices $(\alpha Z, \alpha \mathrm{A}$, $\alpha \mathrm{B}$, and $\alpha \mathrm{C}$ ) connected by a diverse loop region which surround a central acetylated lysine binding site. For the BET subclass of bromodomains, two of these modules are contained within each protein. The large hydrophobic component of this $\mathrm{N}$ acetyl-binding site has made it an attractive target for drug discovery efforts.

A 2012 study assessed the potential druggability of bromodomains using available structural information in the PDB using the software package SiteMap to analyze the size and solvent accessibility of each hydrophobic pocket, resulting in a set of predictions on overall druggability [41]. During the analysis, the authors attempted to rank the potential druggability of the bromodomain family in relation to other known target types. The BET (type I) subclass is predicted to be one of the most amenable to identifying small-molecule inhibitors. A number of type II bromodomains also fell into this category. The higher scoring predictions were driven, in part by the presence and positioning of key residues which define the size and access of the hydrophobic region, leading to a larger and exposed surface area. 
Many of the other bromodomains showed lower predicted druggability scores suggesting lower hit rates from potential screening campaigns. Although classed as difficult to drug, the authors do describe these scores as consistently higher than many "featureless" protein-protein interaction sites. In contrast, the highest scoring results were deemed to be as druggable as kinases, such as aurora kinase, or Hsp90. Since this paper, an increasing number of small-molecule inhibitor chemotypes have been described for bromodomains with examples published covering many areas of the bromodomain phylogenic tree, including examples classified by the Vidler paper as difficult (e.g., BAZ2B).

\subsubsection{BET Bromodomain Inhibitors}

\section{BET Bromodomain Target Introduction}

BET inhibitors have been demonstrated to play oncogenic roles by mechanisms impacting proliferation, cell cycle, and apoptosis in a number of hematological and solid tumor models. More specifically, BET inhibitors are able to downregulate MYC expression in a range of preclinical models of myeloma and myeloid leukemia through multiple mechanisms [42, 43]. BRD2, BRD3, and BRD4 are implicated in the maintenance of aberrant chromatin states in a range of hematological cancers including leukemia, lymphomas, and myelomas. Of the BET family members, the function of BRD4 has been most thoroughly studied. BRD4 regulates RNA pol II-mediated elongation and transcription by directly interacting with mediator complex and pTEFb (positive transcription elongation factor complex) [21, 44, 45]. Additionally, recent literature has shown direct BRD4 interactions with transcription factors, including NF- $\kappa \mathrm{B}, \mathrm{ER} \alpha, \mathrm{p} 53$, TWIST, and GATA-1, across a range of cancer types [46-48]. Thus, bromodomains such as BRD4 serve as a node between disease-relevant transcription factors and the transcriptional machinery. In the context of oncology, BRD4 mediates combinatorial interactions among acetylated histones, transcription factors, nuclear proteins, and the transcriptional machinery, allowing translation of the epigenetic code into RNA synthesis. Amplifications of c-MYC are found in approximately $30 \%$ of prostate cancers, and MYC overexpression is proposed to play a role in prostate cancer initiation [49]. BET inhibitors have also been shown to be effective against NUT midline carcinoma (NMC), a particularly rare epithelial tumor driven by fusions of the NUT protein with either BRD3 or BRD4 genes [50].

BET bromodomain inhibitors have also been implicated in a number of non-oncology indications, due to their ability to selectively regulate specific inflammatory cytokines, including interleukins $1 \beta, 6,17,21$, and 23 [51, 52]. 


\section{BET Tool Compounds}<smiles>CC1=C(C)C2C(c3ccc(Cl)cc3)=N[C@@H](CC(=O)OC(C)(C)C)c3nnc(C)n3C2S1</smiles>

1

JQ-1<smiles>CCNC(=O)C[C@@H]1N=C(c2ccc(Cl)cc2)c2cc(OC)ccc2-n2c(C)nnc21</smiles>

4

GSK525762

(I-BET762)<smiles>COc1ccccc1S(=O)(=O)Nc1ccc2c(c1)CN(C)C(=O)N2</smiles>

$$
2
$$

PFI-1

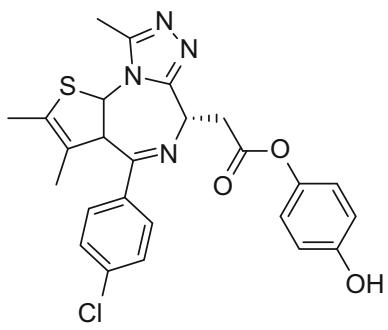

5

MK08628

(OTX-015)<smiles>COc1cc2c(cc1-c1c(C)noc1C)ncc1[nH]c(=O)n([C@H](C)c3ccccn3)c12</smiles>

3

I-BET151

6

CPI-0610

A 2009 patent application from Mitsubishi Tanabe Pharma Corporation [53] first described the thienotriazolodiazepine scaffold as inhibitors of BRD enzymes. Multiple variations on this tricyclic scaffold chemotype were published by other groups in the following years. In late 2010, Filippakopoulos, Knapp, and Bradner et al. discussed the identification and characterization of JQ-1 (1), as a pan inhibitor of the BET domain proteins, and provided evidence of both in vitro and in vivo activity against NUT midline carcinoma [54]. Compound 1 binds in the $N$-acetyllysine binding cleft between the hydrophobic ZA and BC loops. Mimicking the natural $N$-acetyl substrate, the triazole ring of 1 interacts via hydrogen bonding with the conserved asparagine residue (Asn120 in BRD4, Asn429 in BRD2). The group demonstrated the cellular activity of $\mathbf{1}$ in displacing BRD4 from nuclear chromatin and inducing squamous differentiation and growth arrest in BRD4-dependant carcinomas such as NMC. This activity against BRD4-dependent cancer was extended in vivo with activity against multiple patient-derived xenograft models [55-58]. 1 was made available to researchers through the Structural Genomics Consortium (SGC) and was featured in a number of additional studies highlighting 
the effect of BET inhibition against a range of c-MYC driven cancer types including Merkel cell carcinoma (MCC) [55] and leukemia.

BET tool compound PFI-1 (2), described in 2013, was the result of a collaboration between Pfizer and the SGC $[59,60]$. The compound was identified by alpha assay displacement of histone $\mathrm{H} 4$ peptide acetylated at lysine residues $\mathrm{K} 5, \mathrm{~K} 8, \mathrm{~K} 12$, and $\mathrm{K} 16$ with an $\mathrm{IC}_{50}=220 \mathrm{nM}$ and $\mathrm{IC}_{50}=98 \mathrm{nM}$, for BRD4(1) and BRD2(2), respectively. ITC was performed producing $\mathrm{K}_{\mathrm{D}}$ determinations in good alignment with the $\mathrm{IC}_{50}$ values, confirmed similar affinities for other members of the BET family, and showed selectivity over a number of other bromodomains. 2 binds to the conserved NAc interacting residue Asp140 through the carbonyl and nitrogen of the dihydroquinazoline-2-one ring system and also forms a water-mediated hydrogen bond with Tyr98. On-target cellular effects of this inhibitor were demonstrated by the observation of diffusion of GFP-BRD4 from chromatin using a FRAP binding assay in BRD4-dependent NMC cell line. Furthermore, $\mathbf{2}$ inhibits proliferation in a subset of leukemic cells which contained known oncogenic rearrangements in the MLL locus. Concurrent with 2 treatment, the authors observe a significant downregulation of Aurora kinase B. Aurora B activity is linked with c-MYC function [61], and the authors observed that 2 was strongly synergistic with the potent pan-Aurora kinase inhibitor VX-680.

$\mathbf{2}$ has a good selectivity profile against a range of GPCRs, ion channels, and kinases. Rat PK showed the compound to have moderate clearance in good agreement with values predicted by rat liver microsomes and moderate oral bioavailability $(38 \%)$ believed to be driven at least in part by limited solubility in the gut.

I-BET151 (3) represents a second distinct chemotype discussed here which are potent inhibitors of BET bromodomain proteins BRD2, BRD3, and BRD4 [6264]. The other chemotype is represented by the clinical compound $\mathbf{3}$, discussed later in this chapter. 3 is a potent inhibitor of BRD2, BRD3, and BRD4 with reported $\mathrm{pIC}_{50}=6.3 \pm 0.39,6.6 \pm 0.26$, and $6.1 \pm 0.22$, respectively. From a cancer context, the compound was shown to have efficacy in models of murine MLL-AF9 and human MLL-AF4 leukemia. The GSK team has also proposed the mode of action of this compound to act, at least in part, by inhibition of transcription at some key genes including $B C L 2, c-M Y C$, and $C D K 6$ via displacement of BRD3/BRD4, $\mathrm{PAFc}$, and SEC components from chromatin.

\section{BET Inhibitor Clinical Compounds}

Clinical investigation of bromodomain compounds in cancer indications has focused primarily with BET inhibitor space. Development of clinical candidates has been rapidly pursued by a number of companies with a total of seven compounds in early stages of clinical development by late 2015 (Table 2). Many of the chemotypes are related to the original diazepine ring system. Listed below are a few examples of these clinical candidate compounds. 
Table 2 BET bromodomain inhibitors in the clinic

\begin{tabular}{l|l|l}
\hline Drug name & Status & Indication \\
\hline MK-8628/OTX015 & Phase I/II & Glioblastoma \\
\hline INCB054329 & Phase I/II & Advanced malignancies \\
\hline ABBV-075 & Phase I & Advanced cancers \\
\hline BAY-1238097 & Phase I & Neoplasms \\
\hline BMS-986158 & Phase I & Solid tumors \\
\hline CPI-0610 & Phase I & Lymphoma \\
\hline TEN-010 & Phase I & AML and solid tumors \\
\hline
\end{tabular}

GlaxoSmithKline disclosed iBET-762 (4) contemporaneously with $\mathbf{1}$ in 2010 as an equipotent inhibitor of BRD2/BRD3/BRD4 (32-42 nM, FRET assay), binding in both of the tandem bromodomains of BET [65]. Compound $\mathbf{4}$ was identified by a combination of diversity and targeted screening for activators of an ApoA1luciferase reporter in HepG2 cells, highlighting molecules which were able to upregulate reporter gene activity [66]. This was also the primary cellular assay employed in the lead optimization toward 4 . The compound was shown to have a favorable selectivity profile against a range of bromodomains including BAZ20B, SP140, ATAD2, CREBBP, and PCAF [67]. Beyond bromodomains, iBET-762 displayed a favorable profile against a CEREP type panel of other targets $(<30 \%$ when screen at $10 \mu \mathrm{M}$ ) and was not mutagenic in the Ames test. This chemical series shares the benzodiazepine scaffold with $\mathrm{GABA}_{\mathrm{A}}$ allosteric modulators such as alprazolam, and selectivity against GABA receptor activity was achieved through meta- or para-substitution on the pendant phenyl ring. 4 has a free fraction of approximately $20 \%$ and shows moderately low levels of clearance coupled with oral bioavailability $(\% \mathrm{~F})$ around $50 \%$ in primates and dogs. Higher levels of clearance were observed in rodents. The compound is currently in clinical trials against NUT midline carcinoma [68] and relapsed and refractory hematological malignancies [69]. The GSK team has also proposed prostate cancer as a potential indication for 4 [42]. To date, there have been no disclosures on tolerability of the compounds in these early clinical studies. GSK has also shown potential for use of BET inhibitors in non-oncology indications such as rheumatoid arthritis [65].

An additional GSK-derived BET inhibitor (GSK2820151) was disclosed in late 2015 as the company initiated a phase I dose escalation study of subjects with advanced or recurrent solid tumors [70]; however, no additional information is currently available.

OncoEthix SA, a small Swiss biotechnology company, in-licensed OTX-015 (5) in 2012 from Mitsubishi Tanabe. Following initiation of clinical development, OncoEthix was purchased by Merck in 2014. In the process Merck captured the potential first-in-class oral BET inhibitor. The compound (subsequently named MK-8628-001) is a potent binder to BRD2/BRD23/BRD24 with EC50s ranging from 10 to $19 \mathrm{nM}$ and competitive inhibition against acetylated histone 4 [71]. This 
binding activity translated to a compelling in vitro response with a range of $<500 \mathrm{nM}$ $\mathrm{gIC}_{50}$ values in a range of leukemia, lymphoma, and multiple myeloma cell lines [72]. 5 initially induced cell cycle arrest with $\mathrm{G}_{1}$ accumulation and a decrease in S-phase and was predominantly cytostatic but induced apoptosis after longer periods of treatment. The compound was shown to reduce the levels of BRD2 and BRD4 but induced little reduction of BRD3 levels during compound treatment of AML and ALL cells. Observed reductions in $c-M Y C$ expression and protein levels are a broad class effect and attributed to the BRD4 reduction [73, 74]. The BRD2 reduction is also believed to contribute to the antiproliferative effect by inhibition of the Stat5 activity. Indeed, 5 negatively regulated transcripts encoding members of the NF-kB, TLR, and JAK/STAT signaling pathways. In an additional recent study in B-cell lymphoid tumors, $\mathbf{5}$ has shown antiproliferative activity in multiple in vitro and in vivo experiments. Several synergies were also observed in combination with many known anticancer drugs covering a range of mechanisms of activity (Table 3).

A phase Ib dose-escalation study of $\mathbf{5}$ in patients with hematological malignancies was recently discussed in which samples from a total of 81 patients were collected [75]. The data derived from this study suggested the compound has an absorption rate constant $\left(k_{\mathrm{a}}\right)=0.73 \mathrm{~h}^{-1}$, volume of distribution $=71 \mathrm{~L}$, and clearance $=8.5 \mathrm{~L} \mathrm{~h}^{-1}$. When coupled with the relative half-life of around $5.7 \mathrm{~h}$, the group suggested that steady state is reached after only 2 days with dosing at $80 \mathrm{mg}$ QD. Thrombocytopenia was considered to be the principal dose-limiting toxicity in this study.

Constellation Pharmaceuticals first disclosed their clinical candidate BET inhibitor CPI-0610 (6) in 2015 [76, 77] with early details on tolerability from a phase I study. In this early study, CPI-0610 was reportedly dosed QD for 14 days of a 21-day cycle, and the compound has an apparent oral clearance rate of $15.1 \pm 7.0 \mathrm{~L} \mathrm{~h}^{-1}$ with a pharmacokinetic half-life of $10.7 \pm 3.5 \mathrm{~h}$. During the early part of the study, expression of the BET target gene CCR1 was suppressed at

Table 3 Combination effects observed with MK-8628/OTX015 and oncology therapies with a range of targets

\begin{tabular}{l|l|l}
\hline Drug & Combination drug MOA & Activity \\
\hline Everolimus & mTOR & Strong synergy \\
\hline Ibrutinib & BTK inhibitor & Strong synergy \\
\hline Idelalisib & PI3K-delta inhibitor & Synergy \\
\hline Vorinostat & Class I and II HDAC inhibitor & Synergy \\
\hline Rituximab & Anti-CD20 mAb & Synergy \\
\hline Decitabine & DNMT inhibitor & Synergy \\
\hline Lenalidomide & Immunomodulant & Synergy \\
\hline Romidepsin & Class I HDAC inhibitor & Moderately additive \\
\hline Bendamustine & DNA modifying & Moderately additive \\
\hline Doxorubicin & DNA intercalation & Moderately additive \\
\hline
\end{tabular}


multiple doses suggestive of a pharmacodynamic effect in patients. The principal finding from dosing this compound appeared to be a reversible dose-dependent thrombocytopenia. Despite the early stages of the trial, some anti-lymphoma activity was observed with compound $\mathbf{6}$ treatment.

\subsubsection{Other Bromodomain Tool Compounds}

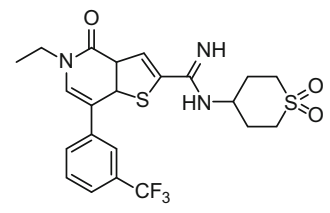

7 I-BRD9<smiles>COc1ccc(Oc2cc3c(cc2NS(=O)(=O)c2ccc(OC)c(OC)c2)n(C)c(=O)n3C)cc1</smiles>

11

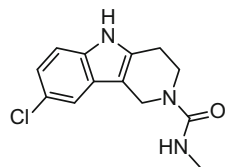

8
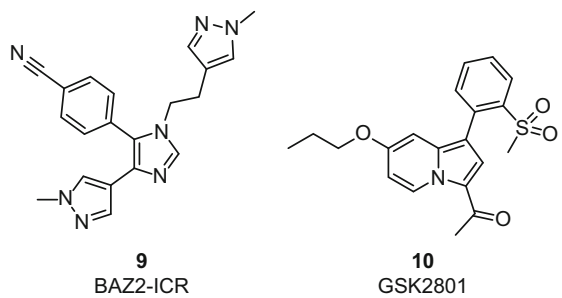

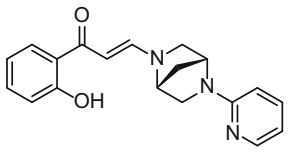

13 PFI-3

A broad collaborative group from GSK and the Universities of Cambridge and Strathclyde published the first example of a series of compounds exemplified by tool compound I-BRD9 (7) [78]. Compound 7 was developed from an initial screen against a GSK internal library. The compound has potent inhibitory activity against BRD9 $\left(\mathrm{pIC}_{50}=7.3\right)$ and displays $>700$-fold selectivity over the remaining BET family. Furthermore, 7 is $>70$-fold selective over a panel of 34 additional human bromodomains and exhibited no activity $<5 \mu \mathrm{M}$ on a range of additional biological targets including receptors, transporters, ion channels, and kinases when compared to BRD4. Endogenous protein binding in cells was confirmed using a NanoBRET assay. A comparative treatment of 7 and pan BET inhibitor 4 was performed in Kasumi-1 cells which highlighted a number of genes downregulated by the selective 7 inhibitor not seen in the 4. Many of these genes have roles in cancer and immunology pathways. No data has been provided on the ADME/PK performance of 7 . 


\section{BAZ Inhibitors}

The bromodomain adjacent to zinc domain (BAZ) family of proteins was assessed by Vidler et al. to be one of the most difficult bromodomain members to drug. The reason for this low druggability scoring derived from a lack of a deep, enclosed hydrophobic pocket seen in other higher scoring bromodomains such as the BET family, despite having an open active site. The BAZ family members are ubiquitously expressed proteins with conserved domain structure including PHD and bromodomain main tail reader motifs [79]. A frequent target of hit-finding efforts, BAZ2A is a component of the nucleolar remodeling complex (NoRC) and a member of the "imitation switch chromatin remodeling complexes" or ISWI [80], which plays a role in regulating the expression of noncoding RNAs and in the formation and repressive heterochromatin. High expression of BAZ2A has been reported in prostate cancer and has been suggested to be a prognostic marker of metastatic potential. Little is known in contrast, about the biological role of BAZ2B; however, high expression levels are associated with poor outcome of pediatric B-cell lymphoblastic leukemia (B-ALL) [81].

Members of the Structural Genomics Consortium (SGC) based at the University of Oxford recently published details of a number of small-molecule fragments which can inhibit BAZ2B [82]. The compounds were identified after an AlphaScreen assay with BAZ2B and a histone H3 peptide acetylated at lysine 14 . Compound 8 inhibits $\mathrm{BAZ2B}$ with an $\mathrm{IC}_{50}=9 \pm 4 \mu \mathrm{M}$, thus suggesting the $\mathrm{BAZ}$ family may indeed be druggable. Additional screening and assessment of binding via differential scanning fluorimetry (DSF) on BRD2-BD1 and CREBBP suggested that $\mathbf{8}$ may have some degree of selectivity for BAZ2B.

Following this initial study, the SGC in collaboration with researchers at the Institute of Cancer Research (ICR) published data on BAZ2-ICR (9), a nanomolar BAZ2A/B inhibitor [83]. After identification of a low micromolar hit, again by AlphaScreen assay, the hit was found to occupy the bromodomain binding site with a phenyl ring positioned in the site normally occupied by the acetylated lysine. After installation of a pyrazole group to act as an acetyl-lysine mimetic and a second (tethered to a flexible alkyl chain) to retain an intramolecular $\pi$-stacking interaction, tool compound 9 was identified. The molecule is an inhibitor of BAZ2A and BAZ2B (130 and $180 \mathrm{nM}$, respectively) in the biochemical readout and was shown to displace BAZ2 proteins from chromatin in living cells in a fluorescence recovery after photobleaching (FRAP) assay. Compound 9 also has potential as an in vivo tool compound having moderate clearance and good oral bioavailability $(F=70 \%)$. 
An additional BAZ2A/B inhibitor chemotype was contemporaneously discovered by a different group of SGC members at Oxford University, this time in collaboration with GSK [84]. The fruit of this research was probe compound GSK2801 (10), which inhibits BAZ2A and BAZ2B with an $\mathrm{IC}_{50}=400 \mathrm{nM}$ and $\mathrm{IC}_{50}=420 \mathrm{nM}$, respectively. Compound 10 was selected as a tool compound as it represented the best selectivity SAR profile against BRD4(1) and BRD9 (>50fold). Like 9, 10 effectively demonstrated the expected cellular mode of action using a FRAP-based assay and has a pharmacokinetic profile suitable for use as an in vivo tool compound.

\section{TRIM24 Inhibitors}

Two independent studies from the SGC/University of Oxford (compound 11) [85] and the MD Anderson Cancer Center IACS-9671 (12) [86] published tool compounds with similar chemotypes as dual inhibitors of bromodomains TRIM24 and bromodomain-PHD finger protein (BRPF). Identification of precursors for both of these compounds was enabled by an AlphaScreen of TRIM24 using a histone H3K23Ac peptide. In both studies, the compounds were shown to interact with TRIM24 and BPRF 1 and BPRF2 in a cellular context but do not discuss the ADME or PK properties of these chemotypes.

\section{SWI/SNF Inhibitor}

The switch/sucrose non-fermentable (SWI/SNF) is a multi-subunit chromatin remodeling complex which has gathered considerable attention as a potential target in cancer research as various subunits are mutated or lost at high frequency in human tumors. The complex contains one of two mutually exclusive helicase/ ATPase catalytic subunits, SMARCA2 and SMARCA4. Together with core and regulatory subunits, SMARCA2/SMARCA4 couples ATP hydrolysis to the perturbation of histone-DNA contacts. In recent studies using genetic loss-of-function (LOF) approaches, three groups have independently identified SMARCA2 as an essential gene in SMARCA4-deficient lung cancer [87-89] proposing a synthetic lethality therapeutic approach. However, it was unclear whether small-molecule inhibitors of the SMARCA2 bromodomain or ATPase domain would be the most pharmacologically relevant target [90].

A group of researchers from MD Cancer Center and Pfizer published results on a potent inhibitor of both SMARCA2 and SMARCA4 PFI-3 (13) [28]. Despite producing a potent and cell-permeable probe molecule capable of displacing ectopically expressed, GFP-tagged SMARCA2-bromodomain from chromatin, the compounds do not display antiproliferative phenotypes. The authors further confirmed via inducible RNAi and cDNA complementation (with bromodomain and ATPase-dead constructs) that the antiproliferative phenotype is driven by the ATPase region and not the bromodomain. 


\section{Writers: Methyltransferases}

\subsection{Introduction and Structural Biology}

Collectively known as protein methyltransferases (PMTs), some 96 proteins have been suggested to perform methylation on histones and other proteins. These PMTs catalyze the transfer of a methyl group from the cofactor S-5'-adenosyl-L-methionine (SAM) to arginine $(\mathrm{R})$ or lysine $(\mathrm{K})$ residues and are further classified based upon this function as RMT or KMTs (Fig. 5a, b). Based upon their protein sequences, phylogenic trees of the KMT and RMT families were created and are shown in Fig. 5c.

\subsection{Arginine Methyltransferases}

\subsubsection{Tool Compounds}

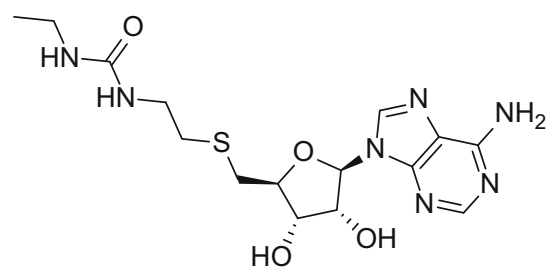

14

DS-437

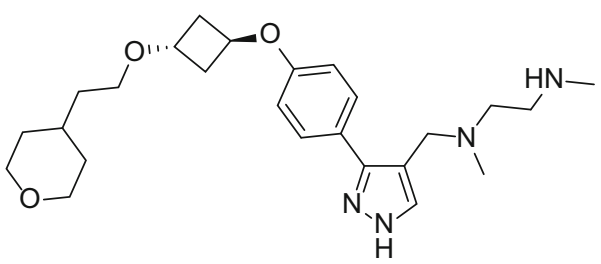

16

EPZ020411<smiles>O=C(NC[C@H](O)CN1CCc2ccccc2C1)c1cc(NC2COC2)ncn1</smiles><smiles>O=C(NCC(=O)N1CCCC1)Nc1ccc2cnccc2c1</smiles> 


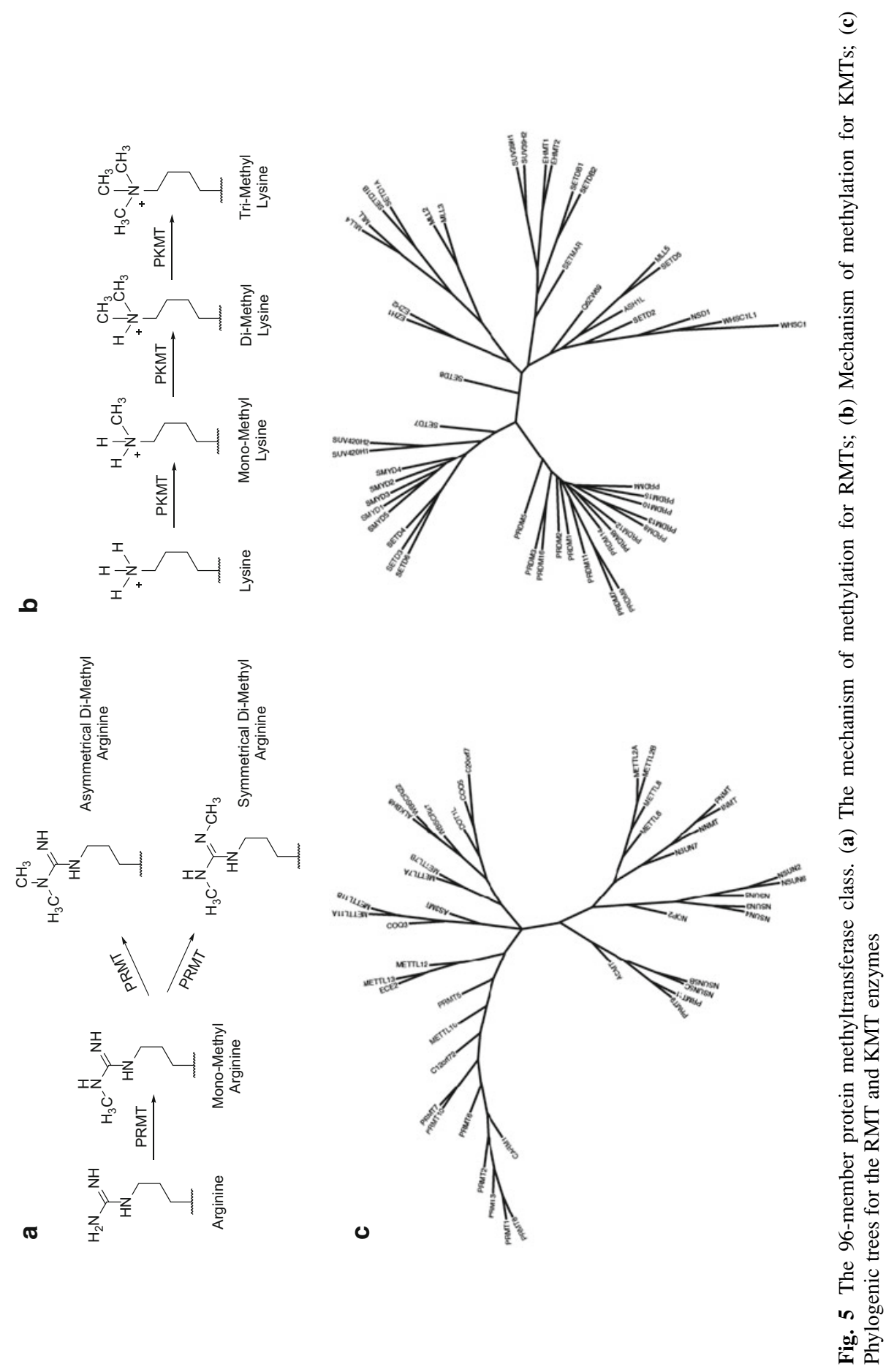




\section{PRMT5}

PRMT5 is a type II arginine methyltransferase capable of performing mono- and symmetric dimethylation of arginine residues on a range of nuclear and cytoplasmic protein substrates and is currently believed to be the predominant enzyme for symmetric dimethylation. PRMT5 may play an important role in tumorigenesis and is upregulated in several human malignancies [91-97]. The mechanism behind the cell-transforming capabilities of PRMT5 has been postulated to have roles in cell death, cell cycle progression, and cell growth and proliferation but is still under investigation [98]. Whether PRMT5 drives tumorigenesis by direct signal transduction, regulation of gene expression or by some other mechanism is generally unknown; however, recent studies highlight a dependency on PRMT5 as part of the spliceosomal machinery with Sm proteins, particularly for MYC-driven tumors [99].

In almost every biological role hypothesized for PRMT5, the enzyme functions as part of a binary complex with the protein MEP50 (methylsome protein 50). MEP50 was initially identified as a WD40 repeat protein, a family of proteins known to play roles in scaffolding, recognition, and activation by mediating interactions between binding partners and substrates. The roles of MEP50 and PRMT5 are inextricably linked as improvements in stability and activation with the complex have been reported by multiple groups. The PRMT5/MEP50 complex was crystallized in 2012 by researchers at Eli Lilly [100], highlighting the tight binding between the two proteins and further confirming the functional role of the complex.

In 2015, the SGC published a nucleoside-derived inhibitor DS-437 (14) with biochemical inhibitory activity against both PRMT5 and PRMT7 $\left(\mathrm{IC}_{50}=5.9\right.$ and $6.0 \mu \mathrm{M}$, respectively) [101]. The compound is a SAM competitive inhibitor of PRMT5 and displayed inhibition of known PRMT5 spliceosomal protein substrates $(\mathrm{SmB}, \mathrm{SmD} 1,3)$ between 10 and $30 \mu \mathrm{M}$. This study was followed a month later by a publication from researchers at Ohio State University who described that PRMT5 is overexpressed following EBV infection and drives B-lymphocyte transformation [102]. In this paper, the group described a small molecule identified from a virtual screen on a PRMT5 homology model. This compound was capable of blocking EBV-driven transformation; however, no biochemical $\mathrm{IC}_{50}$ against PRMT5 was reported. At the same time, Epizyme described the first small-molecule inhibitor of PRMT5 to demonstrate in vitro and in vivo activity in mantel cell lymphoma [103]. Identified from an HTS screen of PRMT5/MEP50, this group identified a hit compound which inhibits PRMT5 enzymatic activity with an $\mathrm{IC}_{50}=325 \mathrm{nM}$. 
This compound was optimized with a blend of structure- and property-guided design [104] leading to tool compound EPZ015666 (15), an orally bioavailable compound which demonstrated robust tumor growth inhibition and a corresponding inhibition of SmD3 in multiple MCL xenografts. Having observed a reduction of cytoplasmic PRMT5 substrates, the group was unable to observe levels of any histone target, H2AR3, H3R8, or H3R4, after tool compound treatment. Whether or not this observation is a limitation of available antibody reagents to visualize subtle histone methyl mark changes or that the observed antitumor effects are driven by other PRMT5-mediated mechanism remains to be determined. In addition to the tool compound, GSK announced in 2016 initiation of the first in human clinical study with an RMT inhibitor with compound GSK3326595 (EPZ015938), a product from their alliance with Epizyme [105].

\section{PRMT6}

PRMT6 is reported to play a role in a variety of processes including DNA repair [106] and regulation of cell cycle [107]. Its overexpression in several cancer types has linked the enzyme to bladder, lung [108], and prostate cancers [109] and melanoma [110]. EPZ020411 (16) is a PRMT6 inhibitor from Epizyme described in 2015, which demonstrates selectivity of $\geq$ tenfold for PRMT6 over other RMT enzymes such as PRMT8 and PRMT1 [111]. The tool compound displays an $\mathrm{IC}_{50}=10 \mathrm{nM}$ against PRMT6 and elicits a concomitant reduction in PRMT6induced H3R2 methylation in A375 cells $\left(\mathrm{IC}_{50}=0.637 \pm 0.241 \mu \mathrm{M}\right)$. PK evaluation of the tool compound showed it to have modest CL $(19.7 \pm 1.0 \mathrm{~mL} / \mathrm{min} / \mathrm{kg})$ and good bioavailability $(F=65.6 \pm 4.3 \%)$ after subcutaneous administration.

\section{PRMT3}

PRMT3 plays a role in ribosomal biosynthesis and has been implicated in inactivation of certain tumor suppression pathways. The first PRMT3 inhibitor, resulting from a high-throughput screen of 16,000 chemical compounds and confirmed by SPR, was reported by the Jin Lab in 2012. The resultant $2.5 \mu \mathrm{M}$ inhibitor was found to have an allosteric binding mode, noncompetitive with peptide and cofactor, and was successfully co-crystalized in conjunction with the SGC to afford a $2 \AA$ crystal structure bound to PRMT3 (3SMQ). Compound 1 was determined to be a selective inhibitor of PRMT3 over KMTs and the more closely related RMTs PRMT1, PRMT4, and PRMT8; this selectivity is hypothesized to result from the novel binding mode of the inhibitor. The initial compound was deemed not appropriate for cellular assays, but further SAR led to a related piperidine-containing compound SGC707 (17) with $31 \mathrm{nM}$ inhibitory activity and improved drug properties $[112,113]$. 


\subsection{Lysine Methyltransferases}

\subsubsection{DOT1}

\section{Target Introduction}

Disrupter of telomeric silencing-1-like (DOT1L), the human homolog of the yeast protein DOT1, is a lysine methyltransferase responsible for catalyzing the methylation of H3K79 [114-117]. The enzyme can catalyze the mono-, di-, and trimethylation in a distributive (i.e., non-processive) manner [118, 119]. Since its discovery, this PMT has been implicated in transcriptional regulation, cell cycle regulation, and DNA damage response. Knockout studies in mice have shown that mouse DOT1L plays an essential role in embryonic development, hematopoiesis, cardiac function, and the development of leukemia [120]. It is the involvement of DOT1L enzymatic activity in leukemogenesis driven by a subset of MLL (mixedlineage leukemia) fusion proteins which has engendered interest in the possibility of targeting DOT1L as a potentially viable target in oncology.

MLL is a genetically distinct form of acute leukemia that constitutes over $70 \%$ of infant and around $10 \%$ of adult acute myeloid leukemias (AMLs). In every case, MLL represents an aggressive form of leukemia and is generally associated with a poor prognosis and with poor response rates from existing therapies. MLL is characterized by a translocation of the MLL gene on chromosome 11q23 $[121,122]$. Patients harboring these translocations typically have an overall 5-year survival rate of just $10-20 \%$.

Under normal conditions, the MLL gene encodes for a SET domain PMT which catalyzes the methylation of H3K4 at specific gene loci [123, 124], with gene localization determined by specific interactions with recognition elements outside the SET domains. However, under the disease-linked translocations, the H3K4directed SET domain is lost, and the remaining MLL protein is fused to a range of protein partners, including members of the AF and ENL family of proteins. With these alternate fusion partners, the MLL protein is capable of recruiting DOT1L, promoting methylation of $\mathrm{H} 3 \mathrm{~K} 79$ in place of $\mathrm{H} 3 \mathrm{~K} 4$, leading to enhancement expression of leukemogenic genes including HOXa and MEIS1 [125, 126].

\section{Structural Biology}

Full-length human DOT1L consists of some 1,537 amino acids, but less than 400 of these are shared with its yeast homolog DOT [114]. The crystal structure of DOT1L containing its methylating co-factor SAM was published in 2003 by Min et al. [118]. DOT1L is unique within the KMT family as it contains no SET domain and the conformation of SAM within the active site is closer to that of RMT members [127]. Despite this similarity in SAM-binding conformation, no data has emerged that suggests DOT1L is capable of methylation of arginine substrates. 
Tool Compound Studies<smiles>CC(C)N(C)CC1OC(n2cnc3c(N)ncnc32)[C@H](O)[C@@H]1O</smiles><smiles>CN(CCCNC(=O)Nc1ccc(C(C)(C)C)cc1)CC1O[C@@H](n2cnc3c(N)ncnc32)[C@H](O)[C@@H]1O</smiles><smiles>CC(O)N(CCCNC(=O)Nc1ccc(C(C)(C)C)cc1)CC1O[C@@H](n2ccc3c(N)ncnc32)[C@H](O)[C@@H]1O</smiles>

20

EPZ004777<smiles>CCCN(CCCCC(N)C(=O)O)CC1OC(n2cnc3c(NC)ncnc32)[C@H](O)[C@@H]1O</smiles>

22<smiles>CC(C)N(CCCNC(=O)Nc1ccc(C(C)(C)C)cc1)CC1OC(n2cc(Br)c3c(N)ncnc32)[C@H](O)[C@@H]1O</smiles>

21

SGC0946

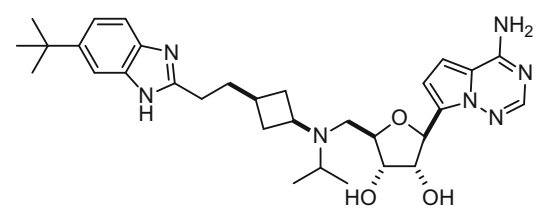

23<smiles>CC(O)N(CC1O[C@@H](n2cnc3c(N)ncnc32)[C@H](O)[C@@H]1O)C1CC(CCc2nc3ccc(C(C)(C)C)cc3[nH]2)C1</smiles>

Epizyme initiated a DOT1L drug discovery program based upon analogs of the co-factor, SAM. This effort produced the small-molecule probe compound EPZ004777 (20) in 2011 [128]. Starting from amino nucleoside compound 18 $\left(K_{\mathrm{i}}=12,000 \mathrm{nM}\right)$, a series of alkyl chain extensions delivered increasing potency which provided $19\left(K_{\mathrm{i}}=13 \mathrm{nM}\right)$. Further modifications of the nucleoside lead to tool compound 20 which inhibits DOT1L with a $K_{\mathrm{i}}=0.3 \mathrm{nM}$ [129] [Basavapathruni, 2012]. Throughout the evolution of SAR, the binding mode of the nucleoside portion of the molecule remained consistent with SAM. However, the increasing amino alkyl chain region of the analogs promotes a distal conformational change not present in the apo form of the enzyme. This conformational alteration creates a hydrophobic pocket to accommodate the substituted phenyl ring of $\mathbf{1 9}$ and 
20. The new inhibitor-induced conformation also led to an increase in inhibitor residence time [129].

Compound 20 has selectivity against a number of additional PMT enzymes, (1,280-fold against PRMT5, >100,000 fold against other tested PMTs). H3K79 mono- and dimethyl levels were reduced in multiple cell lines including MLL fusion-expressing MV4-11 cells. Methyl mark depletion was observed after $24 \mathrm{~h}$ but required up to 4 days to reach maximum levels. In addition, 20 blocks expression of leukemogenic genes and results in selective killing of cells bearing the MLL gene translocation. MV4-11 cells transduced with pMMP-LucNeo retrovirus [130] were used to establish a genetically engineered disseminated leukemia model. 20 was dosed to this model via subcutaneous infusion over a period of 14 days resulting in a dose-dependent increase in median survival.

The Structural Genomics Consortium followed the publication of $\mathbf{2 0}$ with a report highlighting data on analog SGC0946 (21) with similar biochemical potency against DOT1L and selectivity profile. 21 did, however, report an approximate tenfold improvement in cellular potency as demonstrated by reduction of $\mathrm{H} 3 \mathrm{~K} 79 \mathrm{~m} 2$ levels in MCF10A cells. This increased cellular activity was attributed to an improvement in cellular membrane permeability coupled with the extended residence time observed with this chemical series.

Additional chemotypes have been described in the patent applications as inhibitors of DOT1L. Epizyme has also created non-ribose-related analog of 20 [131]. Baylor University has also published on related nucleoside-containing compounds with significant biochemical activity by extending substitution of the iPr group in $\mathbf{2 0}$ with the design tenant of gaining access to the lysine binding channel, compound 22 a key component of this design [132, 133]. Finally, Kainos Medicine Inc. also published a set of adenosine mimic compounds similar to compound 23 [134].

\section{Clinical Development}

Only one DOT1L-targeted compound has progressed to clinical trials. Described in 2013 by Epizyme Inc. [135], pinometostat (EPZ-5676) (24) entered phase I clinical evaluation in 2012 in advanced hematologic malignancies, including acute leukemia with rearrangement of the MLL gene [136]. An additional pediatric trial commenced in 2014 as a large number of pediatric leukemias carry the MLL rearrangement. 24 inhibits DOT1L with a $K_{\mathrm{i}}=0.08 \mathrm{nM}$, displays a residence time of over $24 \mathrm{~h}$, and is over 37,000-fold selective over other PMTs tested. In a cellular context, the compound inhibits H3K79me2 and proliferation of MV4-11 cells with an $\mathrm{IC}_{50}$ around $3 \mathrm{nM}$. In non-MLL rearranged cells, methyl mark inhibition was also observed but with minimal effect on proliferation. PK parameters for $\mathbf{2 4}$ were consistent with earlier tool compounds, and continuous IV infusion was required for dosing in the clinical setting. From a multispecies study, the excretory and metabolic pathways for EPZ-5676 were determined to be similar with low renal excretion of both parent $\mathbf{2 4}$ and related metabolites 
[137]. Fecal excretion of the parent $\mathbf{2 4}$ and a major metabolic mono-hydroxylation of the tertiary butyl group accounted for the majority of drug-related elimination. The group has explored alternative forms for dosing with at least one report investigating subcutaneous, extended-release formulations.

As of the end of 2015 [138], dose-proportional PK was observed with rapid attainment of steady-state plasma concentrations (Css) on day 1 of treatment. Css correlated with inhibition of global H3K79me2 in PBMCs. H3K79me2 ChIP-Seq demonstrated 24 induced reductions in methylation at MLL-r target genes HOXA9 and MEIS1 (median inhibition $=61 \%$; range $=13-91 \%$ ) and was consistent with DOT1L inhibition. Fifteen of 51 patients had either marrow response or resolution of leukemia cutis or leukocytosis/differentiation. Two complete responses and one partial response were observed in patients shown to have rearrangement of the MLL gene. The compound was deemed to have good tolerability with only nine patients displaying grade $\geq 3$ non-hematologic-related toxicities including hypophosphatemia $(n=1)$, decreased ejection fraction $(n=3)$, or elevated transaminases $(n=1)$. Overall adverse events were limited to $>15 \%$ of patients.

24 has shown synergistic antiproliferative activity in MLL-r cell lines when in combination with a number of AML standard of care therapies, hypomethylating agents, and other epigenetic targeted therapies currently in early stages of clinical development including LSD-1 and bromodomain inhibitors [139].

\subsubsection{EZH2}

\section{Target Introduction}

The extensive protein machinery involved in chromatin remodeling works in concert to modify nucleosome-DNA contacts resulting in the selective gene transcription required for myriad biological processes from maintenance of pluripotency to DNA damage repair and tumor suppression. Vulnerabilities can emerge with change-of-function mutations of EZH2 in the context of the larger PRC2 complex. Additionally, in EZH2 antagonizing, chromatin remodeling systems, such as SWI/SNF and BAP1, loss-of-function mutations can lead to H3K27 hypermethylation - by relieving the antagonistic activity of the systems with regard to PRC2 function - and alternate disease states. The known genetic alterations and their resulting cancer indications are listed in Table 4 [140].

There is a growing support in the literature that EZH2 is central to tumor biology, but the oncogenic role of EZH2 may be dependent on the cellular context. For example, EZH2 loss of function in bone marrow cells has been attributed to oncogenesis of T-cell leukemia [145, 146]. Additionally, evidence of tumor suppressor functions has emerged in myeloid malignancies where EZH2 inactivation is correlated with poor prognosis [147]. Recent publications have also highlighted $\mathrm{EZH} 2$ inactivation as a promoter of drug resistance in multiple myeloma [148]. Interestingly, EZH2 has been implicated in methylation of STAT3 which 
Table 4 Genetic alteration or disease associations of the EZH2-containing PCR2 complex

\begin{tabular}{|c|c|c|c|}
\hline Target & Genetic alteration or disease association & Indication & References \\
\hline \multirow[t]{6}{*}{$\begin{array}{l}\mathrm{PRC} 2 \\
(\mathrm{EZH} 2)\end{array}$} & $\begin{array}{l}\text { Heterozygous activating mutations occurring } \\
\text { at Y641, A677, and A } 687 \text { that result in } \\
\text { hypertrimethylation of } \mathrm{H} 3 \mathrm{~K} 27\end{array}$ & Lymphoma & \multirow[t]{6}{*}{ [141-144] } \\
\hline & $\begin{array}{l}\text { Deletion of miR-101 leads to } \mathrm{EZH} 2 \\
\text { overexpression }\end{array}$ & Prostate cancer & \\
\hline & Deletion of INI1 leads to EZH2 dependency & $\begin{array}{l}\text { Malignant rhabdoid } \\
\text { tumors }\end{array}$ & \\
\hline & $\begin{array}{l}\text { Deletion of SMARCA4 leads to EZH2 } \\
\text { dependency }\end{array}$ & $\begin{array}{l}\text { Malignant rhabdoid } \\
\text { tumor of the ovary }\end{array}$ & \\
\hline & $\begin{array}{l}\text { Heterozygous mutations at Y153, H694Y, and } \\
\text { P132S }\end{array}$ & Weaver syndrome & \\
\hline & Deletion of BAP1 leads to EZH2 dependency & Mesothelioma & \\
\hline
\end{tabular}

contributes to tumor progression in glioblastoma [149], but other studies have shown that prolonged EZH2 knockdown may lead to an increased level of proliferation and tumor progression [150]. It is clear that $\mathrm{H} 3 \mathrm{~K} 27 \mathrm{me} 3$ is a mark that plays a significant role in cell fate determination during development. For example, in the mammalian B-cell life cycle, an unnatural trimethylated state of H3K27 arrests the germinal center in the dark zone, preventing differentiation and resulting in aberrant proliferation as opposed to transition to the light zone and either further B-cell maturation, if needed, or alternative apoptosis in a healthy immune system [151]. Preclinically, it has been observed that treatment with EZH2 inhibitors leads to enrichment of B-cell maturation gene sets, suggesting that EZH2 inhibition could result in a cancer cell differentiation event that would lead, in time, to selective apoptosis [152]. One of the advantages of a selective EZH2 inhibitor is that only cells with a genetic addiction to EZH2 activity should be affected by global reduction in $\mathrm{H} 3 \mathrm{~K} 27 \mathrm{me} 3$ which could enable dosing of therapeutics up to efficacious exposures without deleterious side effects from general cellular toxicity. As compounds move into more advanced clinical trials and additional preclinical experiments are enabled with active tool compounds, we expect our collective understanding of the role of EZH2 in cancer to continue to expand in the near future.

\section{The EZH2 Mutant Hypothesis}

Point mutations at or near the catalytic domain of EZH2 have been found in approximately $20-30 \%$ of germinal center diffuse large B-cell lymphoma and follicular lymphoma populations of non-Hodgkin lymphoma patients. All identified mutated residues, such as Y641, A677, and A687 (nomenclature referring to EZH2 variant NM_001203247), are change in function mutations that present in patients heterozygously and function to alter substrate specificity. WT EZH2 is effective at increasing methylation from H3K27 to H3K27me and from H3K27 to H3K27me2, but catalytic efficiency wanes progressively with each successive methylation 
event. The result is low concentrations of H3K27me 3 in most WT cell populations. In the case of heterozygous Y646X mutants $(\mathrm{X}=\mathrm{F}, \mathrm{N}, \mathrm{S}, \mathrm{C}$, or $\mathrm{H})$, however, the mutant enzyme is complementarily more efficient at catalyzing the final methylation step, leading to high levels of H3K27me3 at the expense of H3K27me2 when combined with the WT enzyme activity (Fig. 6). The A677G and A687V mutants, by contrast, lead to hypertrimethylation irrespective of the presence of WT EZH2 and as a result have been referred to as "super-EZH2" enzymes [153].

EZH2 became an attractive target in the personalized medicine era, in part due to the fact that the mutational status of the patient population could be used as a biomarker to potentially predict successful outcome upon treatment with smallmolecule inhibitors. Based upon preclinical data in human-derived xenograft models, patients bearing specific EZH2 mutations may benefit greatly from treatment with EZH2 inhibitors. To date, only a small number of mutant-bearing NHL patients have been dosed with EZH2 inhibitors; hence, this hypothesis remains to be tested fully. It is worth noting, however, that NHL patients without EZH2 mutation have also benefitted from treatment with EZH2 inhibitors suggesting that mutational status is not a requirement for treatment efficacy.

\section{SWI/SNF Complex Machinery Impacts PRC2 Gene Expression}

DNA transcription and repair is also governed by ATP-dependent chromatin remodelers. One such remodeler is the switch/sucrose non-fermentable factor, or SWI/SNF complex, a 14-protein megastructure that regulates transcription by mobilizing nucleosomes. As part of the network of interconnected gene expression machinery, SWI/SNF acts as a tumor suppressor in part by antagonizing the activity of the PRC2 complex in normal cells [154]. However, inactivating subunit mutations of SWI/SNF are found in $\sim 20 \%$ of human cancers and cancer cells [155]. When biallelic mutations of the SWI/SNF complex lead to inactivation by

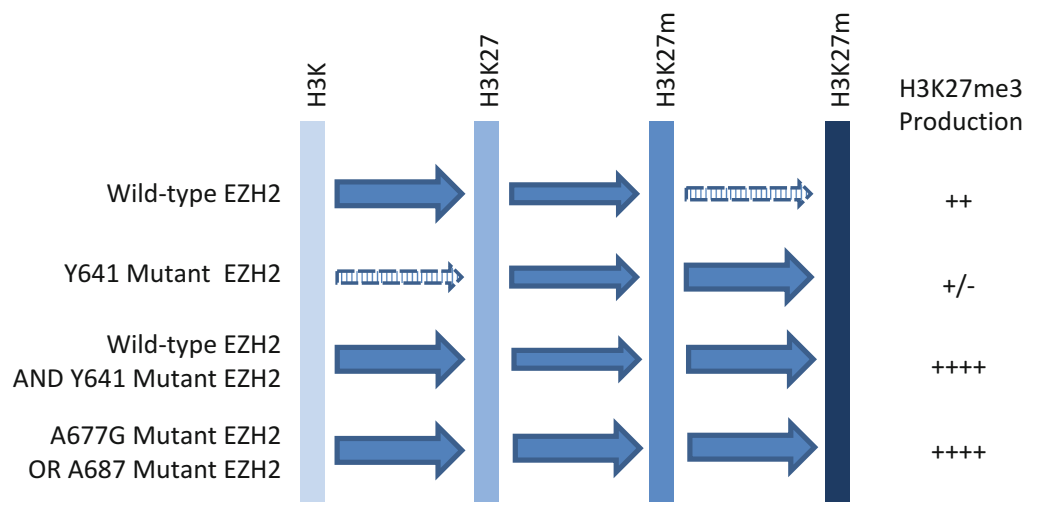

Fig. 6 Predicted effect of mutational status on H3K27 methylation. Wild-type EZH2 produces little H3K27me 3 on its own but, in conjunction with a heterozygous mutation, leads to a hypermethylated state 
loss of certain specific subunits like INI1 or SMARCA4 or SMARCA2, there can be resultant activation of the PRC2 complex and increased EZH2 activity. This, in turn, leads to the potentiation of stem cell programs through repression of target genes that drive to a subset of solid cancers such as malignant rhabdoid tumors (MRT) [156]. MRT is particularly devastating, being characterized by an approximate 12-month median post-relapse survival rate, and although it can occur in adults, it most often affects young children. A related disease, known as malignant rhabdoid tumor of the ovary (MRTO), occurs in young women and constitutes a particular form of ovarian cancer (it is also referred to as small cell carcinoma of the ovary, hypercalcemic type or SCCOHT) [157]. MRTs have no current standard of care treatment and respond poorly to conventional chemotherapies, but are hypothesized to be treatable with EZH2 inhibitors, which would block the associated activation of the PRC2 complex that attends SWI/SNF loss of function mutations.

\section{Loss of BAP1 Leads to EZH2-Dependent Transformation}

BAP1 is a tumor suppressor that exists in a multi-protein complex with ASXL1 (another tumor suppressor). The two proteins act in concert to remove posttranslational, monoubiquitination marks from histone H2A lysine 119 (H2AK119Ub). BAP1 loss-of-function mutations are found in almost half of all mesothelioma. Significantly, like the SWI/SNF relationship, loss of BAP1 is associated with increased H3K27me3 levels and increased expression of EZH2 protein. Recently, Levine's group described the interplay between BAP1 and EZH2, where the myeloid cell-stimulating effects induced by loss of BAP1 function are ameliorated by concomitant loss of EZH2. They also showed that mesothelioma cells resulting from BAP1 loss-of-function mutants are sensitive to treatment with EZH2 inhibitor EPZ011989 (see Sect. 3.2.2.1) [144]. BAP1 represents a second example of how a loss-of-function mutation in one complex is pathobiologically equivalent to a gain of function of EZH2, thus leading to the potential for targeted intervention with an enzyme inhibitor. This discovery was revealed through careful analysis of The Cancer Genome Atlas (TCGA) which shows that EZH2 mRNA expression was increased in mesothelioma tumors compared to healthy tissue. Continued preclinical studies are ongoing to reveal additional potential indications that share this common phenotype.

\section{The Glucocorticoid Receptor Antagonist Synergy Hypothesis}

A great majority of relapsed or refractory NHL patients have previously been treated with a standard of care cocktail of drugs known as R-CHOP (rituximab, cyclophosphamide, hydroxydaunorubicin, Oncovin, and prednisone). This treatment can result in a 60-70\% complete response rate, but approximately one third of 
responding patients relapse, and the recurrent lymphoma can exhibit resistance to a wide array of cancer drugs. The efficacy of EZH2 inhibitors in heavily pretreated patients or as combination therapy in frontline treatment is a subject that has attracted significant attention from companies pursuing EZH2 inhibitors for the market.

Preclinical assessment of EZH2 inhibitors with each of the active ingredients in CHOP individually revealed an intriguing synergy between EZH2 inhibition and glucocorticoid receptor antagonists like prednisolone, the active metabolite of prednisone [158]. Moreover, although mutant cell lines are known to be sensitive to EZH2 inhibition in vitro, EZH2 wild-type GCB lymphoma cell lines (e.g., DOHH2 and OCI-LY19) have demonstrated limited response to these compounds. However, when prednisone is co-administered with EZH2 inhibitors, a marked antiproliferative effect is seen, even in the insensitive DOHH2 WT line with a tenfold improvement over prednisone alone. The potential for this combination to render refractory $\mathrm{GCB}$ lines more sensitive to $\mathrm{EZH} 2$ inhibitors is intriguing and could lead to new frontline therapy combinations including EZH2 inhibitors.

\section{Structure and Function}

EZH2 is the primary catalytic center of the multiprotein polycomb repressive complex 2 (PRC2), the only known complex responsible for distributive methylation of lysine 27 on the histone $\mathrm{H} 3$ subunit (H3K27). PRC2 catalyzes transformation from the native lysine to the mono-, di-, and trimethylated forms, using S-adenosyl methionine (SAM) at each step as the methyl-donating co-factor. $\mathrm{EZH} 2$ acts as an epigenetic regulator because the trimethylated form of H3K27 (H3K27me3) induces a heterochromatic state that is transcriptionally repressive, blocking programmed gene expression [159].

The closely related EZH1 has a lower catalytic activity as a component of PRC2 but is highly related to EZH2 with $96 \%$ homology in the active SET domain. Unlike $\mathrm{EZH} 1$, however, EZH2 is found primarily in rapidly dividing cells like stem cells and is found to be overexpressed in a broad range of cancer types.

Until recently, structural information on the SET domain active site of EZH2 has been limited. The primary reason for this is that the SET domain is at the junction of three proteins, EZH2, EED, and SUZ12, which comprise the active form of the PRC2. Inactive forms of EZH2 have been successfully crystallized, but all three proteins need to be crystallized together with an $\mathrm{H} 3 \mathrm{~K} 27 \mathrm{me} 3$ peptide truncate and the cofactor SAH to obtain meaningful insight into the catalytic domain of the complex. This was accomplished only recently by a group at UT Southwestern with protein from a heat-tolerant eukaryotic fungus strain Chaetomium thermophilum at $2.3 \AA$ resolution [160]. Through this work, Liu et al. have elucidated the intimate relationship between protein subunits, in particular EED and EZH2, and the autocatalytic nature of $\mathrm{H} 3 \mathrm{~K} 27 \mathrm{me} 3$ in transcriptional repression. 
In Vitro Tool Compound Development
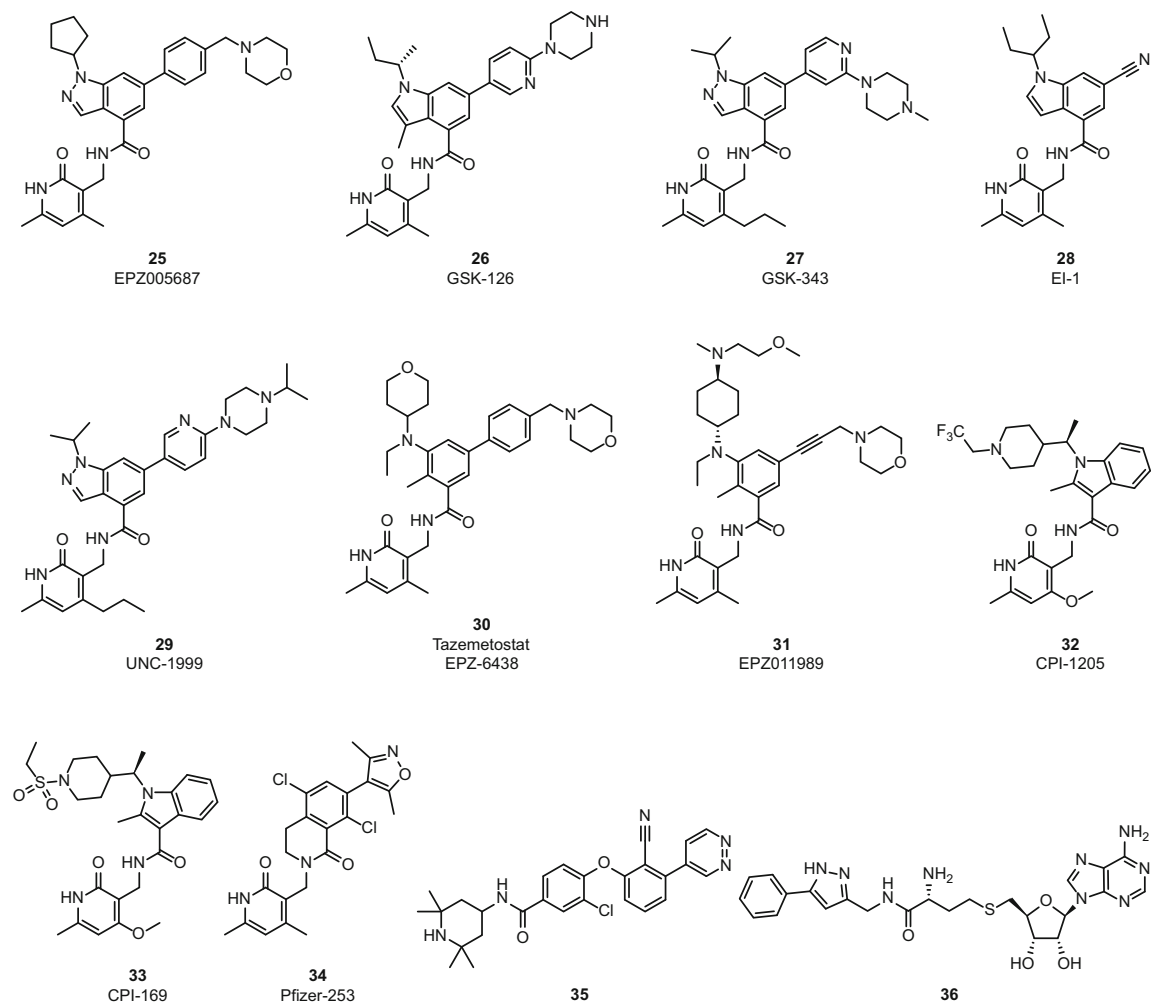

The first series of in vitro tool compounds, developed to establish proof-of-concept antiproliferative effects in cell lines bearing mutant EZH2 isoforms, began to emerge as a group at the end of September 2012 with the publication of Epizyme's

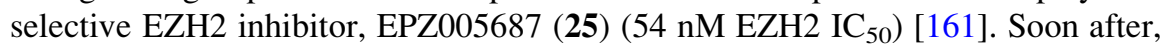
GSK followed with the publication of the compound that would later become their clinical candidate GSK-126 (26) (10 nM EZH2 $\mathrm{IC}_{50}$; see clinical section for further discussion) [162]. This report was closely followed by a second publication from GSK demonstrating the synthesis and EZH2 inhibitory activity of two closely related compounds, GSK-343 (27) (9 nM EZH2 IC 50 ) and GSK-946 [163]. Later, in November of 2012, Novartis reported their preclinical efforts with the disclosure of EI-1 (28) (4 nM EZH2 IC $_{50}$ ) [164], and in April 2013, UNC reported on what they claimed was the first orally bioavailable EZH2 inhibitor, UNC-1999 (29) $\left(<10 \mathrm{nM}\right.$ EZH2 $\left.\mathrm{IC}_{50}\right)$ [165]. No clearance values were reported for the UNC compound so it is unclear whether the compound has the ability to reach efficacious levels of target occupancy upon oral dosing. The most conspicuous conclusion to be drawn from the structures of the first disclosed EZH2 inhibitors is that they all bear a common feature in the 4,6-disubstituted pyridone. With the exception of the UNC 
compound (which is structurally related to compound 27 in the GSK publication from June of the previous year), all of the lead compounds emerged from unique high-throughput screening efforts of commercial or internal diversity chemical libraries.

The cellular activity of each of the in vitro tool compounds has been described in their corresponding papers with a dose-dependent cell killing for each compound in cell lines bearing various EZH2 mutations. The collective data represents an in vitro target engagement proof of concept milestone for these EZH2 inhibitors. Pfeiffer cells which contain the $\mathrm{A} 677 \mathrm{G}$ mutation are particularly sensitive to $\mathbf{2 6}$. In contrast, other Y646X mutant cell lines appear to require dosing at higher concentrations over longer periods of time to achieve a similar response levels.

Although modifications at the 4,6-dimethylpyridone have been made and attempts to replace the pyridone have also been reported, it is accurate to state that the pyridone is an important feature driving potency of EZH2 inhibitors. Without specific structural information of a compound bound in the active domain, we can only speculate that the pyridone amide is acting as both a hydrogen bond acceptor as well as hydrogen bond donor in a network that is optimally oriented by the 4,6-disubstitution pattern.

In a recent medicinal chemistry optimization paper describing clinical candidate EPZ-6438 (30) [166], SAR generated during lead optimization depicts the importance of the orienting methyl substitution on various parts of the EZHZ scaffolds (Fig. 7). The sequential additional of methyl groups on the pyridone ring in compounds 37-42 results in a concomitant increase in inhibitory activity against $\mathrm{EZH} 2$. In addition, blocking of the pyridone amide carbonyl was identified to have a more significant effect on biochemical potency than blocking the amide $\mathrm{NH}$ hydrogen bond donor group. This suggests that it is the amido (41) and not the imido (42) tautomer which confers activity and that the carbonyl oxygen makes a highly favorable interaction with the enzyme. GSK and Constellation teams have reported improved in vitro activity with the 4-N-propyl substitution and 4-methoxy substitution, respectively, but to date, they have not published orally bioavailable analogs bearing these modifications.

A second notable similarity between the in vitro tool compounds presented by each of the major groups in the field is that each contains a bicyclic core with an amide at the 4-position and a highly lipophilic group at the N1-indole or indazole position, an example of which is compound 42 (Fig. 8). In the bicyclic series, very little is tolerated at the N1-position suggesting a highly lipophilic pocket is accessed

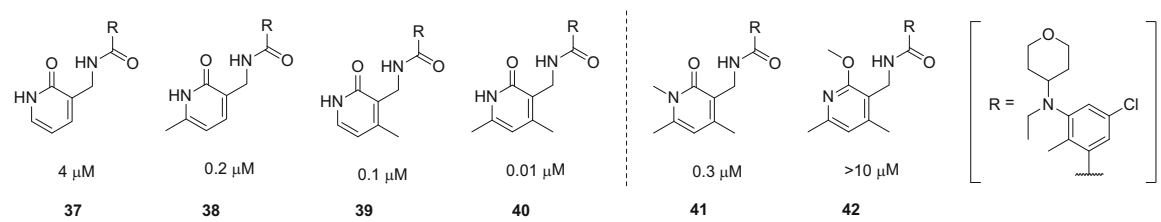

Fig. 7 Impact of pyridone methyl substitution is additive and prefers the free amide carbonyl 

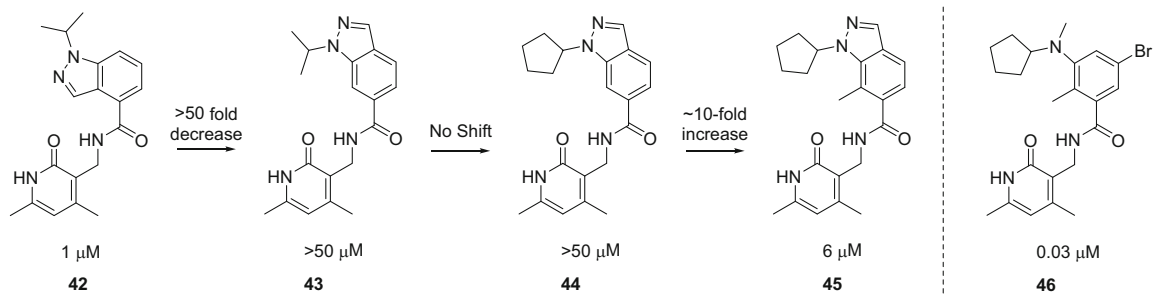

Fig. 8 Identification EZH2 inhibitors with an aryl core scaffold

along this vector. The significance of the orientation of the bicycle with respect to the pyridone-bearing amide was not fully understood until a more thorough investigation of the core revealed that the orientation of the bicycle was not as critical to identifying potency against EZH2, but instead a steric blocking group that forced the amide out of plane is a significant driver of potency (45). Retaining this "magic methyl" and replacing the indole ring for an aryl ring delivered a path to increased potency as shown with compound $\mathbf{4 6 .}$

\section{Next-Generation Tool Compounds}

Since the initial disclosure of in vitro tool compounds, continued compound development has led to a series of more advanced compounds available for preclinical evaluation. EPZ011989 (31) (EZH2 $\left.\mathrm{IC}_{50}=<3 \mathrm{nM}\right)$, reported in early 2015, has good oral bioavailability and demonstrated robust tumor regression in an in vivo xenograft model using the KARPAS-422 cell line implanted into SCID mice, when dosed at $250 \mathrm{mg} / \mathrm{kg}$ twice daily (BID) for 21 days [167]. Low unbound clearance and strong potency against EZH2 make $\mathbf{3 1}$ an ideal in vivo tool compound for probing additional cancer indications that may be susceptible to EZH2 inhibition. Constellation also reported a new indole core chemotype that, although not orally bioavailable, demonstrated a $>10 \mathrm{~h}$ half-life when dosed subcutaneously (SC). In vivo efficacy for compound CPI-22 (32) $\left(\mathrm{EZH} 2 \mathrm{IC}_{50}=2 \mathrm{nM}\right)$ was reported in a similar xenograft model using KARPAS-422 cells at $200 \mathrm{mg} / \mathrm{kg}$ BID with complete tumor regression observed by day 28 . Additionally, Pfizer reported a ring closed scaffold analog PFI-253 (33) that locks the freely rotating pyridine to the core ring with a two- or three-atom tether $\left(\mathrm{EZH} 2 \mathrm{IC}_{50}=<4 \mathrm{nM}\right)$. No biological data was reported in the patent that discloses this new chemotype, but the reduction of H-bond donors in this scaffold suggests the high potential for this new class to deliver oral drug-like molecules. 


\section{Alternative Scaffolds}

One of the most successful attempts to broaden the scope of selective EZH2 inhibitors came from the optimization of another high-throughput screening hit by Constellation Pharmaceuticals [168]. The medicinal chemistry team identified a weakly potent $(51 \mu \mathrm{M})$ EZH2 inhibitor bearing an unusual $2,2^{\prime}, 6,6^{\prime}$-tetramethylpiperine component that, like the pyridine class, demonstrated a SAM competitive phenotype. After an unsuccessful attempt at replacing the diphenyl ether linkage, the terminal phenyl moiety was optimized effectively to deliver a $32 \mathrm{nM}$ inhibitor with potent activity in vitro (34). The cellular antiproliferative activity reached $9.5 \mu \mathrm{M}$ in the KARPAS422 EZH2 Y646N mutant cell line with a concomitant reduction in H3K27me3, but the team appeared unable to deliver a bioavailable compound from this class; hence, no in vivo studies have been reported to date.

Using a strategy similar to that employed in the development of DOT1L inhibitors (Sect. 3.3.1.3), a team at Pfizer, La Jolla, has reported a subset of EZH2 inhibitors designed directly from the reaction product SAH [169]. These nucleoside-based inhibitors, exemplified by compound $\mathbf{3 5}$, demonstrated selective WT and Y646N mutant EZH2 inhibition of 270 and $70 \mathrm{nM}$, respectively. However, no cellular activity was achieved due to perceived permeability limitations.

\section{Clinical Progress with EZH2 Inhibitors}

One of the advantages of EZH2 as a therapeutic target is that incoming patients can be screened to determine their mutational status. This can be used to stratify the treatment population into groups that have or do not have the EZH2 mutation to determine if there is any advantage one way or the other. A second advantage of $\mathrm{EZH} 2$ as a drug target is the specificity of the enzymatic action on this epigenetic mark. This specificity allows for the measurement of EZH2 inhibitors in vivo activity of by monitoring the amount of methylated H3K27 present in tumor or surrogate tissue. This signal loss can be used as a pharmacodynamic (PD) readout of inhibitor activity that can then be correlated with tumor growth inhibition as a less invasive means for measuring in situ target occupancy.

Three compounds had entered phase I human trials for NHL by the end of 2014 and one compound, 30, initiated phase II studies in Europe and the USA in 2015 for NHL and solid tumors, respectively, at the time of writing (Table 5). Significant preclinical evidence supports EZH2 inhibitor activity in specific NHL patient populations for which there exists a significant population that is refractory to current treatment modalities and with a relapse rate suggestive of a clear need for novel therapies. 
Table 5 Clinical studies with inhibitors of EZH2

\begin{tabular}{l|l|l|l}
\hline Drug name & $\begin{array}{l}\text { Target class/ } \\
\text { enzyme }\end{array}$ & Status & Indication \\
\hline Tazemetostat & HMT/EZH2 & $\begin{array}{l}\text { Phase } \\
\text { I/II }\end{array}$ & $\begin{array}{l}\text { Lymphoma, INI-deficient tumors, synovial sarcoma, } \\
\text { SMARCA4-negative tumors }\end{array}$ \\
\hline CPI-1205 & HMT/EZH2 & $\begin{array}{l}\text { Phase } \\
\text { I }\end{array}$ & B-cell lymphoma \\
\hline GSK2816126 & HMT/EZH2 & $\begin{array}{l}\text { Phase } \\
\text { I }\end{array}$ & B-cell lymphoma, follicular lymphoma \\
\hline
\end{tabular}

The Epizyme clinical tazemetostat $\mathbf{3 0}$ was realized through reduction of the bicyclic core of $\mathbf{2 5}$ to a substituted phenyl core as shown earlier. This allowed for property optimization to afford a compound with improved potency and PK properties. 30 was granted the generic name tazemetostat by the WHO in 2015.

The phase I clinical trial with $\mathbf{3 0}$ was designed as a $3+3$ dose escalation study starting at $100 \mathrm{mg}$ BID, dosed orally and targeting a maximum dose of $1,600 \mathrm{mg}$ BID. The trial was open to NHL and solid tumor patients with the primary endpoint and was identified as establishment of the maximum tolerated dose and measurement of the objective response rate. Secondary endpoints were assessment of PK, duration of response, and progression-free survival, to be measured at 8-week intervals.

A phase II was initiated in June 2015 in patients with DLBCL or FL for determination of efficacy and safety in five independent cohorts determined by histology, cell of origin, and EZH2 mutational status. This NHL clinical trial is being conducted in Europe, Australia, Canada, and the USA. In addition, separate phase II trials in patients with advanced solid tumors characterized by INI1 or SMARCA4 negativity or synovial sarcoma were initiated in adults and in pediatric subjects in the USA, Australia, Europe, Canada, and Asia in December 2015.

In January 2014, 26 was progressed to the clinic as a twice weekly intravenous (IV) infusion. The phase I trial was conducted in adult patients with relapsed/ refractory diffuse large B-cell and transformed follicular lymphoma malignancies. The starting dose was $50 \mathrm{mg}$ in water solution, infused over a $2 \mathrm{~h}$ period twice weekly, initially with 3 weeks on and 1 week off in each 28 day cycle, with a maximum tolerated dose cutoff of 3,000 $\mathrm{mg}$ twice weekly. The primary endpoint was to explore the recommended phase II dose in relapsed/refractory germinal center B cell-like diffuse large B-cell lymphoma (GCB-DLBCL) subjects with EZH2 wild-type and EZH2 mutant-positive lymphoma prior to the initiation of an expansion phase. Phase I data are not currently available, but if merited, the expansion phase was projected to have minimally two cohorts based on EZH2 mutation status.

The first example of a pyridone-containing EZH2 inhibitors to be published from Constellation Pharmaceuticals was an advanced compound containing a 4-methoxy6-methylpyridone and a methyl-bearing indole core (CPI-169, 33) appearing in a 
Chemistry \& Biology paper published in 2014 [170]. The compound reported was dosed subcutaneously (SC) in the preclinical models of antitumor activity, but an orally bioavailable analog of $\mathbf{3 3}$ was discovered and has advanced through IND-enabling studies and into the clinic, CPI-1205 (32) [171].

The Constellation phase I human trial was initiated with CPI-1205 (32) in patients with a confirmed diagnosis of a B-cell lymphoma that has progressed in spite of prior treatment [172]. The reported primary outcome measures are frequency of dose-limiting toxicities to be assessed in the first 28 days on study (Cycle 1). Expected enrollment is 41 patients, and the study is predicted to collect all the necessary data by the end of December 2016 .

\section{EZH2 Resistance Mechanisms}

A large body of preclinical data exists that describes the robust effects of EZH2 inhibitors on in vivo xenograft implanted tumor cells bearing EZH2 mutations. However, cancer is highly adaptable, and using an artificial in vitro system, several specific mutations that give certain cell lines the power of resistance to EZH2 inhibitors have been produced [173]. The resistant mutations were identified by a team at Ariad Pharmaceuticals by using forward genetics in the Pfeiffer B-cell lymphoma line bearing an A677G super-EZH2 mutation. After the cells were mutagenized, drug-resistant outgrowths were observed to increase cell viability scores by 1,000-fold posttreatment with EZH2 inhibitors $(\mathbf{2 5}, \mathbf{2 7})$. Sequencing of the resistant lines leads to the discovery that the new mutation was not found in the SET domain, but instead a high-frequency EZH2 Y111D missense mutation, along with several additional low-frequency missense mutations, was identified in the $\mathrm{N}$-terminal allosteric D1 domain. It is notable that at higher doses of inhibitor, no new outgrowth was observed, and Y646F mutant EZH2 lines were shown to be less susceptible to the same mutational resistance.

Importantly, no patients bearing a Y111D mutation have been identified in a clinical setting, and if they do exist, the mutation can be identified by conventional screening and can be treated with alternative therapeutics or higher doses of a EZH2 inhibitor. The authors also suggest that next-generation EZH2 inhibitors could focus on this allosteric site at the EZH2/EED nexus to mitigate acquired resistance mechanisms. Interestingly, targeting this protein-protein interaction was the subject of a recent publication where a cell-permeable stapled peptide with a $K_{\mathrm{D}}$ of $264 \mathrm{nM}$ ( $\mathrm{SN}_{\mathrm{L}} \mathrm{FSSNRQKILERTXILNXEWKQRRIQPV)}$ was shown to reduce H3K27me3 in a MLL-AF9 leukemia cell line [174].

As with many of these types of early mutation studies, there is currently no data confirming that these in vitro selection experiments can or will be recapitulated in a clinical setting. Additionally, as suggested in the paper, higher doses of EZH2 inhibitor may mitigate any specific Y111D mutation. 


\subsubsection{Additional KMT Tool Compounds}
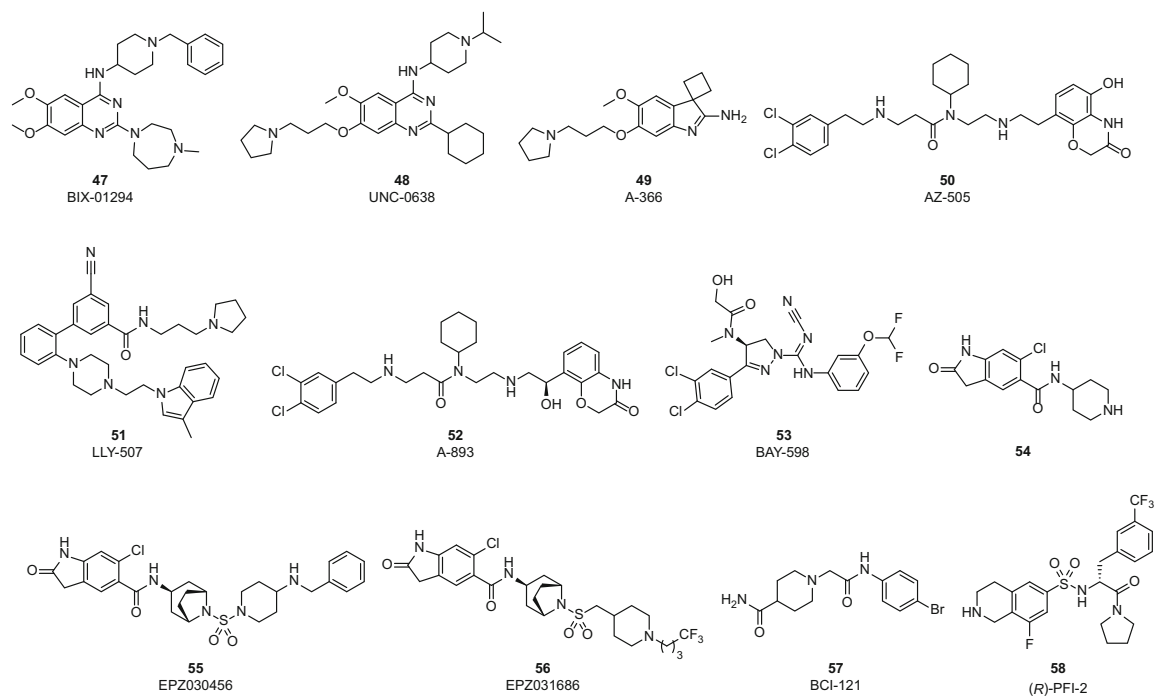

\section{EHMT2}

EHMT2, or euchromatin histone methyltransferase 2, is the gene name for a lysine methyltransferase, also commonly referred to as G9a. As the gene name suggests, the enzyme is associated with the installation of a transcriptional repressive methyl mark. EHMT2 and its close relative EHMT1 (GLP) are known to methylate H3K9 to the mono- and dimethyl state but have also been reported to be able to methylate H3K27 to a lesser degree. EHMT2 belongs to the SET domain-containing family of proteins that include SUV39, SETDB1, and DIM-5 [175]. A number of substrate competitive small-molecule inhibitors have been disclosed by Boehringer Ingelheim (BIX-01294, 47) [176], the University of North Carolina (UNC-038, 48) [177], and Abbvie (A-366, 49) [178].

A number of potential cancer indications have been postulated for EHMT2 including leukemia [179], head and neck cancer [180], esophageal cancer [181], and glioma [182]. Additionally, it was shown recently that EHMT2 appears to also play a role in activation of the $\beta$-globin gene and potentially the induction and of fetal hemoglobin [183-185]. 


\section{SMYD2}

SMYD2 stands for SET and MYND domain-containing protein 2 and is histone methyltransferase that is responsible for monomethylation of $\mathrm{H} 3 \mathrm{~K} 4$ and the addition of a second methyl group to H3K36me, using SAM as cofactor. Despite some localization to the nucleus, active SMYD2 is largely cytoplasmic ( 80\%) and also responsible for methylation of a number of non-histone substrates including p53, HSP90, ER $\alpha$ [186], and PARP1 [187]. Early reports on the cellular function of SMYD2 suggested a possible role in restricting cellular proliferation [188], and SMYD2 has been also implicated as a possible oncogene due to its inactivating methylation of Lys370 of p53, a known tumor suppressor [189]. However, later publications have linked SMYD2 overexpression to poor outcomes in esophageal squamous cell carcinoma [190], gastric cancer [191], and pediatric ALL [192] suggesting the tumor suppression potential of inhibition of this target. Despite the connection to cancer therapy, to date, SMYD2 knockout or inhibition has failed to demonstrate global effects on $\mathrm{H} 3 \mathrm{~K} 4$ or $\mathrm{H} 3 \mathrm{~K} 36$ methylation states. Whether this unique lack of effect is due to the cytosolic localization of the enzyme or another, yet unknown factor is the subject of current research with newly developed tool compounds designed to probe the SMYD2 mechanism. Crystal structures of SMYD2 constructs have been reported with SAM bound (3TG4, 3S7J), SAH and p53 bound (3TG5), as well as with methyltransferase inhibitors sinefungin (3QWW), LLY-507 (51) (4WUY), and A-893 (52) (4YND).

To gain a better understanding of the biology in play when SMYD2 is inhibited in a cellular context, a number of tool compounds have emerged from industry and institutional research programs. The first reported inhibitor, AZ-505 (50), was from AstraZeneca [193]. This compound was developed from a 1.23 million compound HTS campaign using AlphaScreen technology. Compound $\mathbf{5 0}$ is a potent and selective inhibitor of SMYD2 with a biochemical $\mathrm{IC}_{50}$ of $120 \mathrm{nM}$ and $>70$-fold selectivity over SMYD3, the nearest homolog. $\mathbf{5 0}$ was also found to bind in the SET domain of SMYD2 in a peptide competitive and SAM noncompetitive inhibition mode, identified through crystallography efforts. Although no cellular data has been provided for 50, a structurally related compound was recently reported by AbbVie in conjunction with the SGC, 52, that has an additional chiral alcohol that confers a significant increase in biochemical potency $\left(\mathrm{IC}_{50}=3 \mathrm{nM}\right)$. This increased potency, along with the removal of the 5-hydroxy component of the benzoxazinone, is projected to allow for functional activity to be observed via suppression of p53K370 methyl mark in relevant lung carcinoma cell lines [194].

Two additional cell active tool compounds for SMYD2 were also released in 2015. The first compound, produced by a team at Lily Pharmaceuticals, 51, is a potent $(<15 \mathrm{nM})$ inhibitor of SMYD2 with a $>100$-fold selectivity over other tested HMTs [195]. Importantly, however, 51 demonstrated p53K370 methyl mark inhibition with an $\mathrm{IC}_{50}$ of $0.6 \mu \mathrm{M}$ in U2OS cells. Compound $\mathbf{5 1}$ further inhibited the proliferation of KYSE-150 (esophageal) and MDA MB-231 (breast cancer) cell lines in an apparent dose-dependent manner. Despite the increased cellular activity of 51 and 52, neither of these dibasic compounds allow for oral dosing to enable 
in vivo experiments. 53, a compound developed by Bayer and made available to the scientific community by the SGC, is reported to inhibit SMYD2 at $27 \mathrm{nM}$ with good selectivity and cell activity $(<1 \mu \mathrm{M})[196]$.

\section{SMYD3}

SMYD3 plays an important role in the regulation of gene transcription through complexation with the RNA polymerase complex, and overexpression of this histone modifier is linked to enhancement of cell growth. SMYD3 is responsible for $\mathrm{H} 3 \mathrm{~K} 4$ and $\mathrm{H} 4 \mathrm{~K} 5$ di- and trimethylation in the nucleus. It also plays a significant role in the cytoplasmic methylation of MAPK3 (MEKK2) at lysine 260, which may result in an activated MAPK state required for KRAS-driven oncogenesis. Although a full understanding of the mechanism in cancer is still unknown, whether by modification of nuclear or cytoplasmic proteins, SMYD3 activity has been linked to the development and cell survival of breast [197], prostate [198], pancreatic, lung [199], and gastric [200] cancer types. Until recently, validated chemical probes for understanding the biology around SMYD3 and cell viability have been limited. In 2015, however, two new inhibitor chemotypes were introduced. Crystal structures of SMYD3 have been solved with SAM (3MEK), sinefungin (3RUO), small-molecule inhibitor 54 (5CCL), and EPZ030456 (55) bound (5CCM).

The first reported tool compounds to selectively target SMYD3 were developed by Epizyme and reported in 2015 [111]. In vitro tool compounds 55 and $\mathbf{5 6}$ were developed through optimization of a proprietary library hit, compound $\mathbf{5 4}$, by modifying the aminopiperidine into a rigidified sulfonamide with a 4-aminopiperidine or a substituted 4-methylpiperidine tail. The resulting compounds bind in a noncompetitive mode with respect to MEKK2 substrate and mixed-competitive mode versus SAM; however, the compounds do not make many significant interactions with the SMYD3 protein outside of the 6-chloro-2oxoindoline-5-carboxamide head group. Despite this, both compounds have singledigit $\mathrm{nM} \mathrm{IC}_{50}$ values against SMYD3, and this translates to 48 and $36 \mathrm{nM}$ cellular activity in a MEKK2 In-Cell Western (ICW) Assay for $\mathbf{5 5}$ and 56, respectively. Additionally, $\mathbf{5 6}$ was identified as a good candidate for in vivo studies with in vitro ADME (mouse liver microsomes, $24 \mathrm{~mL} / \mathrm{min} / \mathrm{kg}$ and mouse plasma protein binding, 0.53 unbound fraction) and PK studies that demonstrated high oral bioavailability $(>48 \pm 5 \% \mathrm{~F})$ suitable for further efficacy assessment in mouse xenograft models of disease.

A second chemotype identified as a SMYD3 inhibitor was also recently published by an academic group in Bari, Italy [201]. Although proliferation data was provided for BCI-121 (57) in a number of relevant cancer cell lines, the reported data are insufficient to exclude a general cytotoxic effect producing the observed antiproliferative effects. However, the chemotype of $\mathbf{5 7}$ is drug-like and may warrant further examination. 


\section{SETD7}

SETD7 is another SET domain-containing lysine methyltransferase, responsible for monomethylation of $\mathrm{H} 3 \mathrm{~K} 4$ and the resultant transcriptional activation. SETD7 expression is known to be active in skeletal muscle differentiation [202], but the exact role of SETD7 in cellular signaling pathways and the progression of disease is still poorly understood. $(R)$-PFI-2 (58) is a selective small-molecule inhibitor first reported by the SGC in conjunction with Pfizer that has a peptide competitive and SAM uncompetitive binding modality [203]. A $1.9 \AA$ structure of $\mathbf{5 8}$ has been solved in the SETD7 SET domain showing binding in the substrate pocket and hydrophobic interaction of the amide pyrrolidine with the mobile charged methyl group of SAM. Importantly, the $R$-enantiomer has a $\mathrm{K}_{\mathrm{I}}^{\text {app }}$ of $0.33 \mathrm{nM}$ (500 times more potent than the corresponding $S$-enantiomer) and has demonstrated cellular activity in NHLF cells [204]. Using this newly available tool compound, He et al. have reported that SETD7 is implicated in cellular response to oxidative stress through regulation of mitochondrial function. More studies are currently underway with this tool compound to further elucidate the role of SETD7 in disease.

\section{Erasers: Lysine Demethylases}

\subsection{Introduction}

Histone demethylases (HDMs) are a large class of chromatin-modifying enzymes commonly referred to as "erasers" that include peptidyl arginine deiminases (PADI) [205] and the lysine-specific demethylase (KDM) family members 1-8 and their closely related subtypes. Table 6 provides a complete list of the KDMs and their commonly used synonyms [206]. Lysine demethylases can be further stratified into two main groups. The first group contain a Jumonji domain (JmjC) and demethylate through hydroxylation and decomposition of the resulting aminal to formaldehyde employing the cofactors $\mathrm{Fe}(\mathrm{II})$ and $\alpha$-ketoglutarate in the active site (KDMs 2-8). The second group, KDM1 (LSD1), are amino oxygenases that harness molecular oxygen to oxidatively remove methyl marks relying on flavin adenine dinucleotide (FAD) as cofactor and producing formaldehyde and hydrogen peroxide as by-products. While JmjC-containing demethylases are capable of demethylation of trimethylated lysine residues and indeed may prefer Kme3 as substrates [207], they are complementary to the amino oxygenase demethylases which can mechanistically only remove di- and monomethylated marks.

Lysine demethylases have emerged as a critical part to the chromatin remodeling family, linked to the growth and differentiation of embryonic stem cells, ectopic expression, spermatogenesis, and many other biological functions. Additionally, HDMs and the KDM subtypes have been implicated in a number of diseases including leukemia, prostate cancer, squamous cell carcinoma, and X-linked mental retardation. 
Table 6 Lysine demethylases with associated names and synonyms

\begin{tabular}{|c|c|c|}
\hline $\begin{array}{l}\text { Approved } \\
\text { symbol }\end{array}$ & Previous symbols & Synonyms \\
\hline KDM1A & AOF2, KDM1 & KIAA0601, BHC110, LSD1 \\
\hline KDM1B & C6orf193, AOF1 & $\begin{array}{l}\text { FLJ34109, FLJ33898, dJ298J15.2, bA204B7.3, FLJ43328, } \\
\text { LSD2 }\end{array}$ \\
\hline KDM2A & FBXL11 & $\begin{array}{l}\text { KIAA1004, FBL11, LILINA, DKFZP434M1735, FBL7, } \\
\text { FLJ00115, CXXC8, JHDM1A }\end{array}$ \\
\hline KDM2B & FBXL10 & PCCX2, CXXC2, Fbl10, JHDM1B \\
\hline KDM3A & $J M J D 1, J M J D 1 A$ & TSGA, KIAA0742, JHMD2A \\
\hline KDM3B & C5orf7, JMJDIB & KIAA1082, NET22 \\
\hline KDM4A & $J M J D 2, J M J D 2 A$ & KIAA0677, JHDM3A, TDRD14A \\
\hline KDM4B & $J M J D 2 B$ & KIAA0876, TDRD14B \\
\hline KDM4C & $J M J D 2 C$ & GASC1, KIAA0780, TDRD14C \\
\hline KDM4D & $J M J D 2 D$ & FLJ10251 \\
\hline KDM4E & KDM4DL & $J M J D 2 E$ \\
\hline KDM5A & RBBP2, JARID1A & \\
\hline KDM5B & JARID1B & RBBP2H1A, PLU-1, CT31 \\
\hline KDM5C & SMCX, JARID1C & DXS1272E, XE169 \\
\hline KDM5D & $\begin{array}{l}\text { HYA, HY, SMCY, } \\
\text { JARID1D }\end{array}$ & KIAA0234 \\
\hline KDM6A & UTX & \\
\hline KDM6B & JMJD3 & KIAA0346 \\
\hline KDM8 & JMJD5 & FLJ13798 \\
\hline
\end{tabular}

\subsubsection{LSD1 (KDM1A)}

Target Introduction

LSD1 expression is reportedly elevated in a number of human cancers including lung, breast, prostate, and common blood cancers such as AML. Preclinical studies report LSD1 inhibitors having antiproliferative effects in numerous solid and hematological human cancer cell lines in vitro [208]. Moreover, small cell lung cancer (SCLC) LX48 cell line xenograft experiments have demonstrated significant tumor growth inhibition when treated with an oral small-molecule LSD1 inhibitor (vide infra). However, whether LSD1 upregulation or downregulation is beneficial, like histone methyltransferase activity, may be cancer-type specific. It has been observed that LSD1 is downregulated in breast carcinomas which activates the TGF $\beta 1$ signaling pathways and increases the potential for metastasis [209]. Alternatively, high levels of LSD1 are associated, in part, with the likelihood of relapse in prostate cancers post radical prostatectomy [210]. In blood cancer, specific effects of LSD1 inhibitors have been reported on a treatment resistant subset of acute myeloid leukemia (AML) known as acute promyelocytic leukemia (APL) when dosed in conjunction with retinoid standard of care therapy [211]. Others have shown that LSD1 is an essential regulator of leukemia stem cell (LSC) effectors via 
in vivo xenograft models in which cells bearing MLL translocations were targeted over normal hematopoietic stem and progenitor cells (HSPCs) [212]. Recently, Wada et al. have reported LSD1 overexpression appears to correspond with increased incidence of T-cell lymphoblastic leukemia (T-LBL) via upregulation of HoxA family members and increased cellular self-renewal properties [213]. Although the emerging role of LSD1 in cancer appears complex, in order to probe LSD1 biology further, selective cell active inhibitors have been developed to test the above cancer hypotheses.

\section{LSD1 Structural Biology}

Prior to 2004, histone methylation was thought to be unidirectional and therefore a static histone modification installed by histone methyltransferases. LSD1 (KDM1A) was the first histone demethylase to be identified and is unique to the family because it also belongs to the FAD-dependent amine oxidase family that also includes MAO-A and MAO-B. LSD1 is responsible for catalyzing the dynamic demethylation of mono- and dimethylated lysines $\mathrm{K} 4$ and $\mathrm{K} 9$ on the histone $\mathrm{H} 3$ subunit. Cellularly, LSD1 is commonly localized in CoREST repressor complexes that also include HDAC family members, and it is believed that CoREST may be responsible for targeting LSD1 to DNA [214]. Additionally, LSD1 is known to be present in the Mi-2/nucleosome remodeling complex (NuRD) where it can interact with metastasis tumor antigens 1-3 that restrict transcription through interactions of promoter regions [209]. Alternatively, LSD1 can also function as an activator required for expression of androgen receptor (AR) and estrogen receptor (ER) target genes by removing the $\mathrm{H} 3 \mathrm{~K} 9 \mathrm{me} 2$ repressive methyl mark [215]. Other activator complexes that employ LSD1 include the elongation factor RNA polymerase II (ELL) [216] and the MLL epigenetic modifying supercomplex. LSD1, like many related epigenetic enzymes, may perform multiple roles in the maintenance of balanced gene expression, but it has emerged as an important transcriptional factor that is highly expressed in certain forms of cancer, and this makes it an appealing target for drug discovery.

The structure of a truncated LSD1 peptide was solved in 2008 by Chen et al. at the University of Michigan Medical School [217]. The initial structure identified an amine oxidase-like (AOL) catalytic center lined with highly negative electrostatic regions and an N-terminal SWIRM domain responsible for stability and binding interactions with chromatin remodeling machinery. Since that time, crystal structures of covalent inhibitors of LSD1 have also been published [218]. LSD1 is differentiated from the close homolog LSD2, in part, by the presence of a Tower domain which consists of two $\alpha$-helices arranged in a typical antiparallel coiled coil. This substructure emerges from the center of the AOL, contains a repeating pattern of seven amino acid residues, and plays a critical role in mediating the interaction with the CoREST complex. 


\section{Tool Compound Development}

The design and development of LSD1 inhibitors has expanded greatly as research into LSD1 overexpression and role as a potential oncogenic driver in certain cancers has developed. To date, inhibitors can be classified into two different groups, covalent inhibitors modeled after their MAO inhibitor forebears and reversible inhibitors that do not interact directly with FAD upon binding. The benefits and safety of selective, covalent modifiers have been showcased in a number of recent high-profile drug approvals [219]. Irreversible inhibitors commonly lead to enzyme degradation and therefore can lead to a robust time dependent inhibition response at lower concentrations. However, there still remains active interest in developing reversible inhibitors that can differentiate from the irreversible inhibitors in measures of selectivity and safety.

\section{Covalent Inhibitors}

Because MAO inhibitors have long been studied for treatment of depression, a host of compounds known to be active against the FAD-dependent enzymes were used as starting points for LSD1 inhibitor programs. Tranylcypromine [220], an early MAO inhibitor that entered the market for depression in 1960 itself, has inhibitory activity against LSD1 $(214 \mu \mathrm{M})$ although it is 50-fold more potent against MAO-A. Tranylcypromine reacts with FAD via a radical transfer mechanism, leading to an atropaldehyde adduct in MAOs, but due to the nature of the binding pocket of LSD1, the cinnamaldehyde adduct is favored which can further modify FAD to the five-membered intramolecular cycle adduct (Fig. 9) [221, 222].

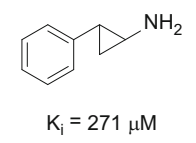

59

Tranylcypromine

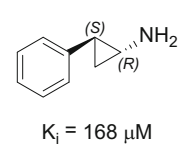

60

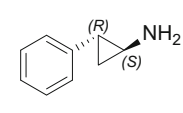

$\mathrm{K}_{\mathrm{i}}=284 \mu \mathrm{M}$

61

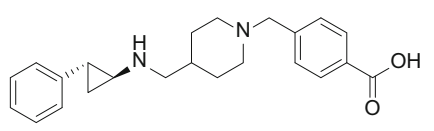

64

GSK-2879552

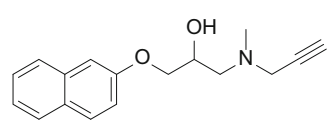

62

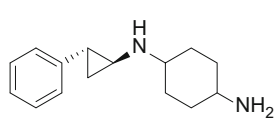

65

ORY-1001

Tranylcypromine-based tool compounds 59-61 are the most commonly reported LSD1 inhibitors in the literature. Early SAR concentrated on the aromatic component of the molecule, and it was found that increased activity correlated with increased lipophilicity on the phenyl ring of the molecule. Additionally, a thorough investigation of the stereochemistry of the cyclopropane showed marked preference for the trans isomer, and furthermore the specific 1S,2R-enantiomer has the strongest 


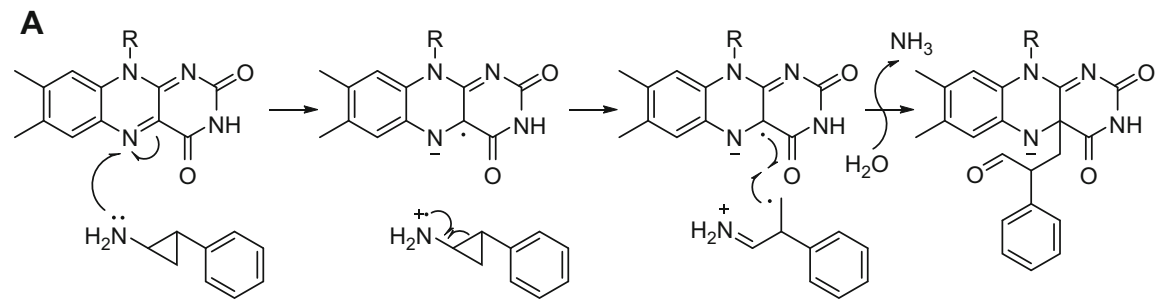

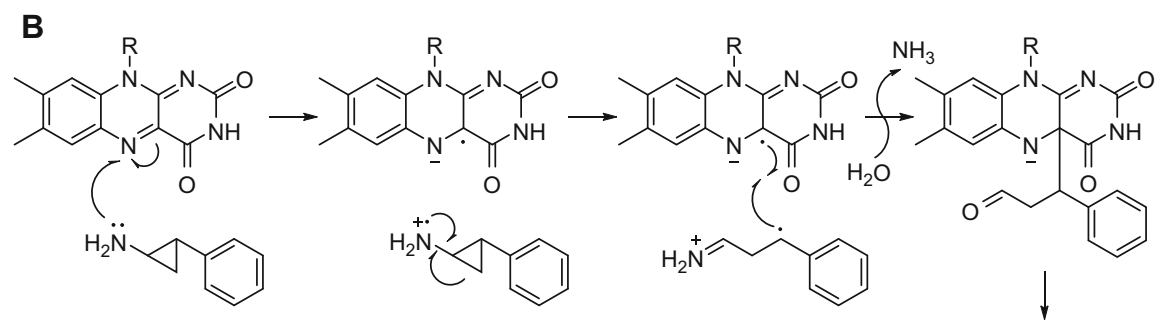

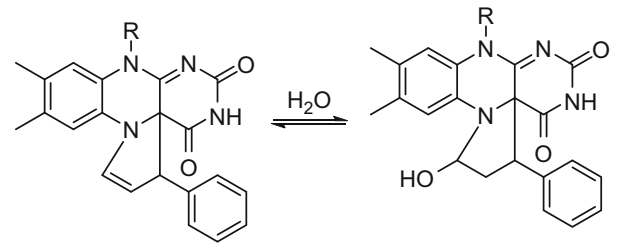

Fig. 9 Proposed FAD modification by tranylcypromine to form (a) atropaldehyde [221] or (b) cinnamaldehyde [222] adducts

binding potential [221]. Subsequently, a new class of compounds with defined stereochemistry and aliphatic substitution on the amine moiety emerged, leading to highly active and more selective inhibitors available for preclinical proof-ofconcept experiments, and have been reviewed [223]. Other MAO inhibitor phenotypes have also been explored as irreversible LSD1 antagonists. For example, Schmitt et al. have reported a pargyline analog, containing a FAD reactive propargyl amine moiety (compound 62), as a $52 \mu \mathrm{M}$ inhibitor of LSD1 with modest selectivity over MAO-A and MAO-B [224]. Additionally, the mechanism-based hydrazinebased derivative Bizine (63), presented by Prusevich et al., has demonstrated LSD1 activity and selectivity over MAO-A (23-fold), MAO-B (63-fold), and LSD2 (>100fold) [225].

While irreversible MAO inhibitors have demonstrated clinical efficacy for depression, they are not widely used today because of their potential for off-target activity and adverse effects. Additionally, certain dietary restrictions limit their utility as a lifetime daily dosing regimen. While the newly designed LSD1 inhibitors 
based on the tranylcypromine scaffold are far more selective than the early FAD-modifying antidepressants, there exists the possibility of a negative side-effect profile. As a result, an active effort to discover more traditional modes of inhibition, including reversible inhibitors, has been initiated by a number of research groups.

Reversible Inhibitors<smiles>CC(C)(C)NCCCCNC/C=C/CNC(C)(C)CCCCNC(C)(C)C</smiles>

$$
\begin{aligned}
& 66 \\
& \text { PG-11144 }
\end{aligned}
$$<smiles>Cc1c(C(F)(F)F)oc2ccc([N+](=O)[O-])cc2c1=O</smiles>

68
Namoline<smiles>O=S(=O)(Nc1nc(-c2ccc(Cl)cc2)cs1)c1cccc(-c2ccccc2)c1</smiles>

72

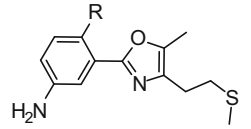

69<smiles>CN=C(NC)N1CCN(Cc2cc(C(=O)OC)cc(C(=O)N3CCN(C(=O)c4ccc(C(=N)N)cc4)CC3)c2)CC1</smiles>

67 CBB-1007<smiles>N/C(CC(=O)Nc1ccccc1O)=N\O</smiles>

70<smiles>C/C(=N\NC(=O)c1cccc(S(=O)(=O)N2CCOCC2)c1)c1cc(Cl)ccc1O</smiles>
71 SP-2528<smiles>[R]CC1CCC=C(Cn2cc(CSC(=S)N3CCN(C(=O)OC(C)(C)C)CC3)nn2)C1</smiles>

73<smiles>Cc1ccc(-c2ncc(OC[C@@H]3CCNC3)cc2-c2ccc(C#N)cc2)cc1</smiles>

Reversible inhibitors of LSD1 are a much more diverse class of compounds that are currently in various stages of preclinical advancement. An excellent, in-depth review published by Mould et al. highlights some of the strengths and weaknesses of the many proposed reversible inhibitors for LSD1 [226]. Some of the earliest purported LSD1 inhibitors reported were polyamine compounds like Progen's PG-11144 (66) which has demonstrated in vitro increases in H3K4me2 in human colorectal cancer cell lines (HCT116) [227]. While related compounds are progressing through the clinic for various tumor types, the cytotoxic effects of this class are difficult to attribute directly to one particular mode of action. $\mathbf{6 6}$ was divested from Progen's portfolio in 2010, and no information on any further development of this compound has been reported. Wang et al. report an analogous diguanidyl compound (CBB-1007, 67) with a rigidified backbone that reached a $2.1 \mu \mathrm{M} \mathrm{IC} \mathrm{I}_{50}$ with a comparable cellular $\mathrm{EC}_{50}$ of $1-5 \mu \mathrm{M}$. Although the compound has low potency, unlike the flexible polyamines 67 , it appears to have direct effects on LSD1.

In more recent reports, a host of compounds have been discovered through highthroughput screening efforts and virtual screens but lack the data to correlate 
biochemical activity with cellular antiproliferative effect required for validation of their identity as specific LSD1 inhibitors. For example, chromenone derivative Namoline $(\mathbf{6 8})$ has weak LSD1 activity $(51 \mu \mathrm{M})$ and is generally cytotoxic in vitro at low concentrations [215]. A second series of phenyl oxazoles as represented by compound 69, based on earlier MAO inhibitors, produced only $10 \mu \mathrm{M}$ biochemical inhibition; however, the reported $\mathrm{IC}_{50}$ curves have high Hill slopes potentially indicative of non-specific inhibition. The amidoxime series presented by Hazeldine et al. (e.g., compound 70) demonstrated $17 \mu \mathrm{M}$ biochemical $\mathrm{IC}_{50}$ against LSD1 but failed to demonstrate consistent cellular increases in H3K4me2 [228]. Lastly, a set of hydrazide inhibitors were discovered by Sorna et al. through virtual modeling of the FAD pocket [229]. Salarius Pharmaceuticals was created to advance the optimized hydrazide compound SP-2509 (71) (13 nM) through IND-enabling studies, but although activity has been reported in a wide number of cancer cell lines, including Ewing's Sarcoma [230], the correlation between biochemical effect and cell activity suggested $\mathbf{7 1}$ activity is a non-specific cytotoxic effect. It is possible that the 2-hydroxybenzylidene)hydrazide moiety is a pan-assay interference structure (PAINS) [231] or cleavage of the hydrazide results in the in situ formation of acyl hydrazines, which are known irreversible and non-specific FAD-dependent enzyme inhibitors.

Despite the high number of unsubstantiated claims, there have also emerged a set of compounds with reliable data that warrant further investigation. These include a series of aminothiazoles (72) generated by fragment library screening at the Institute of Cancer Research in London [232]. Optimized to a top $\mathrm{IC}_{50}$ of $0.4 \mu \mathrm{M}$, this series demonstrated consistent SAR and was found to be reversible with enzyme, selective against MAO-A and translated to expected antiproliferative effects in the expected low-micromolar range. Additionally, a series of dithiocarbamates (e.g., compound 73) was reported by Zheng et al. that show reliable correlation between biochemical and cellular activity but contain a potential cytotoxic chemotype that may limit use of this series as in vivo tool candidates [233]. Lastly, GSK-354 (74), reported in 2013, has the combined features of high biochemical potency with a drug-like core that translates to $1.4 \mu \mathrm{M}$ cellular activity resulting in a FAD noncompetitive reversible compound suitable for in vitro and in vivo testing [234].

\section{Clinical Progress}

As was the case with MAO inhibitors, the first LSD1 inhibitors to enter the clinic possess an irreversible mechanism of action. To date there have been three clinical efforts to treat human cancer in a clinical setting (Table 7). The first trial to initiate was in December 2013 with GSK's tranylcypromine prodrug GSK2879552 targeting AML and SCLC solid tumors in two separate phase I trials $[235,236]$. This was followed closely by Oryzon who entered the clinic at the 
Table 7 Inhibitor of LSD1 in oncology clinical trials

\begin{tabular}{l|l|l|l}
\hline Drug name & Target class/enzyme & Status & Indication \\
\hline ORY-1001 & KDM/KDM1A & Phase I/II & AML \\
\hline Tranylcypromine & KDM/KDM1A & Phase I/II & AML \\
\hline GSK2879552 & KDM/KDM1A & Phase I & AML \\
\hline
\end{tabular}

very end of 2013 to treat refractory acute leukemia. Late in September of 2014, as proposed by Schenk et al. in 2012 [211], racemic tranylcypromine itself is being tested in combination with the all trans-retinoic acid (ATRA) drug tretinoin for relapsed/refractory AML in a small group of patients in Germany and the USA in parallel. The two stand-alone treatments will be discussed in detail below.

GSK-2879552 (64) is an irreversible inhibitor of LSD1 activity that, as a prodrug, is converted to their earlier reported tool compound, with an LSD1 activity of $5 \mathrm{nM}$, through metabolic cleavage of the benzyl benzoic acid component. The compound is reported to be selective for LSD1 inhibition, requiring FAD for activation, and tested negative against a panel of FAD utilizing enzymes, such as MAO-B.

64 entered human testing at the end of 2013 in a phase I trial designed to treat patients with relapsed or refractory small cell lung cancer (SCLC). Additionally, patients were requested to avoid citrus fruits, suggesting a CYP3A4 route of clearance that if blocked could lead to higher than expected drug exposures. The primary objective of the escalation phase is to establish recommended phase II dose (RP2D) and understand any dose-limiting toxicity as guided by the Neuenschwandercontinuous reassessment method (N-CRM). The planned expansion cohorts are expected to enroll patients with relapsed or refractory SCLC to understand efficacy in a larger patient population.

GSK has also initiated a phase I trial with $\mathbf{6 4}$ in patients with acute myeloid leukemia (AML) as of June 2014. Enrollment requirements include classification as relapsed or refractory AML patients with no available standard therapies remaining available for treatment of the disease state. Response rate, maximum tolerated dose, and establishment of a recommended phase II dose were identified as the primary objective outcomes from this study to be evaluated as per the SCLC trial above.

ORY-1001 (65) was originally developed by Oryzon Genomics, in Spain, for the treatment of adult patients with relapsed or refractory acute leukemia [237]. In April 2014, the Oryzon Genomics LSD1 program was licensed by Roche pharmaceuticals before the end of phase I. In August 2015, Oryzon received the first milestone from Roche upon finalization of the multiple ascending dose (MAD) stage of its phase I clinical trial to evaluate the safety, tolerability, and pharmacokinetics of ORY-1001. In November of that same year, Oryzon announced initiation of the expansion of the phase I study to additional sites in Spain, the UK, and France. 


\subsubsection{Additional Tool Compounds}<smiles>O=C(O)c1ccnc(-c2cn(C3CCCN(C(=O)c4ccccc4)C3)nn2)c1</smiles>

75

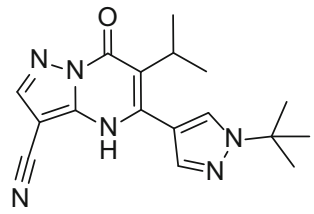

76

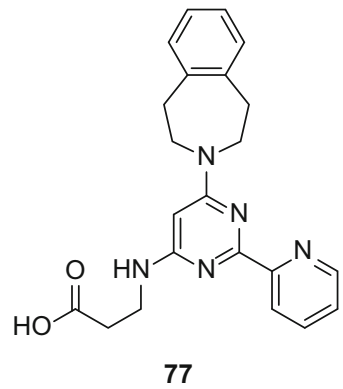

GSK-J1

Although small-molecule inhibitors of Jumonji domain-containing (JmjC) lysine demethylase targets have, until recently, largely been the purview of academic researchers, there has been a significant increase in publications and patents emerging from industry in 2015 [238]. This increase in activity parallels growing evidence that supports the hypothesis that aberrant KDM activity is linked to eukaryotic transcription, treatment resistance mechanisms [239], and the development of physiological disease states. In the subsequent subsections, we describe the four families for which selective small-molecule inhibitors have been described in the current literature.

\section{KDM2 Family}

KDM2A and KDM2B are Fe-dependent lysine demethylases that act upon H3K36 with a preference for the dimethylated substrate H3K36me2. KDM2A activity has been implicated in maintaining centromeric integrity and genomic stability [240, 241] and regulation of NF-kB [242] and has been shown to be overexpressed in certain lung, ovarian, bladder, and esophageal cancer patient samples. Recently, KDM2A has also been reported as a potential tumor suppressor in breast cancer [243]. Structures of human KDM2A are known with the 2-OG co-factor bound (4QWN, 4QX7), with NOG surrogate co-factor (4QXC, 4QXH), and with a smallmolecule inhibitor contributed by the SGC (4URA). This inhibitor was modified using medicinal chemistry approaches into a selective KDM2A inhibitor, 75, with a $>30$-fold separation from its nearest measured homolog KDM5C. Unfortunately, compound $\mathbf{7 5}$ has poor cellular permeability, however, and in vitro experiments with the methyl ester prodrug are planned to be reported in due course [244]. 


\section{KDM5 Family}

KDM5 lysine demethylases were first reported by Klose et al. in 2007 with the discovery of retinoblastoma-binding protein 2 (RBBP2), later renamed JARID1A and KDM5A, as a demethylase of H3K4me3 and H3Kme2 [245, 246]. KDM5B and KDM5C family members would follow, but KDM5A alone has been identified as a prognostic marker for colorectal [247] and lung cancer [248], implicated in cell fate determination and tumorigenesis [249], drug resistance [250], and AML leukemia [251]. To date crystallographic structures of KDM5 family members remain limited with one linked JmjN and JmjC domain construct of KDM5A crystallized with 2-OG (5E6H) and two small-molecule bound structures of KDM5B with pyridine 2,6-dicarboxylate $(5 \mathrm{~A} 3 \mathrm{~W})$ and a 2 -amidomethyl substituted pyridine 4-carboxylate (5A3T). Additional chemotypes have emerged in the patent and literature recently, including potent and cell-active inhibitors from Constellation Pharmaceuticals [252-254]. Compound 76 has shown $<100$ nanomolar $\mathrm{IC}_{50}$ potency and significant selectivity over KDM2B, KDM3B, KDM4C, KDM6A, and KDM7B homologs in biochemical assays. Compound 76 also specifically affected H3K4me3 and H3K4me2 methylation levels in melanoma (M14), breast (SKBR3), and NSCLC (PC9) cells and demonstrated a reduction in the number of drug-tolerant persister cancer cells (DTPs), making it an appealing in vitro tool compound for further exploration of KDM5 family target validation efforts.

\section{KDM6 Family}

KDM6A and KDM6B were identified by Hong et al. in 2007 to be specific lysine demethylases for $\mathrm{H} 3 \mathrm{~K} 27$ preferring trimethylated and dimethylated H3K27 as substrate [255]. Early reports on KDM6 family members suggested a potential role in proliferation and transcriptional changes [256], but KDM6 family members have also been implicated as tumor suppressors [257]. More recently a malespecific homolog KDM6C (UTY) has been reported to be completely inactive in cells [258]. KDM6B structures are known in the apo form (4EZ4), 2-OG bound (2XUE), peptide and NOG-bound (4EZH), and with 2-OG competitive smallmolecule inhibitors 8-hydroxyquinolone (2XXZ) and GSK-J1 (77) (4ASK).

Compound 77 was one of the first reported small-molecule inhibitors reported by industrial researchers [259]. 77 was reported to exploit the 2-OG-binding site, making a bidentate interaction with the catalytic iron center and was shown to be selective over the KDM4 family homologs. This early tool compound was poorly cell permeable as well, but the ethyl ester was used to demonstrate specific cellular activity of KDM6B on pro-inflammatory gene activation in macrophages. This hypothesis was later challenged by a group at EpiTherapeutics who showed in an expanded battery of cellular assay equal potency for the ethyl ester against KDM4C, KDM5B, and KDM6B suggesting that observed gene effects could not specifically be attributed to KDM6 alone [260]. 


\section{Future of Epigenetic Drug Discovery}

The field of epigenetic drug discovery has grown tremendously over the past decade. After the initial HDAC inhibitor approval in 2006, academia and industrial groups have forged countless discoveries in the understanding of the roles of epigenetic mechanisms in oncology. These discoveries effectively initiated the processes which led not only to new drug discovery targets but validated discovery efforts across three entire classes of potential targets focusing on methyl mark addition (writing), elimination (erasing), and acetyl mark recognition (reading). The clinical targets leading the charge in these spaces are arguably the most biologically validated, which undoubtedly promoted them to the top of the list for many groups as they prioritized efforts to identify druggable chemical matter.

With the kinases, novel chemical matter starting points with little or no selectivity across a range of family members eventually morphed into the ability to design selective inhibitors and thus provide extremely powerful tool compounds to further validate biological hypotheses. In contrast, there does not seem to be a promiscuous PMT inhibitor. In fact, many selective chemotypes have been identified for individual targets or highly structurally related members of a particular branch of a phylogenic tree. This feature has brought with it the advantage of rapid evolution of high-quality chemical biology tools to pharmacologically validate targets which appear interesting from siRNA knockdown or genetic knockout studies. As discussed in this chapter, tool compounds effectively cast doubt on the role of a particular epigenetic target in both methyltransferase (SMYD2) and bromodomain (SMARCA2) areas. Going forward, tool compounds will remain a crucial component in enablement of additional epigenetic-focused drug targets, and perhaps newer techniques such as CRISPR can elucidate protein domains to prioritize drug discovery effort [261].

Over the next 10 years, we will see exactly which of the current clinical programs can actually deliver upon the promise of targeted therapies for hard-to-treat cancers such as INI1-negative malignant rhabdoid tumors (EZH2 inhibitors) or NUT midline carcinoma (BRD inhibitors). New epigenetic drugs could also potentially add synergistic benefits to existing standard-of-care oncology treatments and perhaps new transformative immunotherapy regimes as they emerge. Whatever happens, it promises to be an interesting time for medicinal chemists.

\section{References}

1. Maurer-Stroh S et al (2003) The tudor domain "Royal Family": tudor, plant agenet, chromo, PWWP and MBT domains. Trends Biochem Sci 28(2):69-74

2. Camerino MA et al (2013) The structure-activity relationships of L3MBTL3 inhibitors: flexibility of the dimer interface. Med Chem Commun 4(11):1501-1507

3. James LI et al (2013) Discovery of a chemical probe for the L3MBTL3 methyl-lysine reader domain. Nat Chem Biol 9(3):184-191 
4. James LI et al (2013) Small-molecule ligands of methyl-lysine binding proteins: optimization of selectivity for L3MBTL3. J Med Chem 56(18). https://doi.org/10.1021/jm400919p

5. Biel M et al (2004) Design, synthesis, and biological evaluation of a small-molecule inhibitor of the histone acetyltransferase Gcn5. Angew Chem Int Ed 43(30):3974-3976

6. Bowers EM et al (2010) Virtual ligand screening of the p300/CBP histone acetyltransferase: identification of a selective small molecule inhibitor. Chem Biol 17(5):471-482

7. Chimenti $\mathrm{F}$ et al (2009) A novel histone acetyltransferase inhibitor modulating Gen5 network: cyclopentylidene-[4-(4'-chlorophenyl)thiazol-2-yl)hydrazone. J Med Chem 52 (2):530-536

8. Tsukada Y-I et al (2006) Histone demethylation by a family of JmjC domain-containing proteins. Nature 439(7078):811-816

9. Klose RJ, Kallin EM, Zhang Y (2006) JmjC-domain-containing proteins and histone demethylation. Nat Rev Genet 7(9):715-727

10. Chang B et al (2007) JMJD6 is a histone arginine demethylase. Science 318(5849):444-447

11. Han G et al (2012) The hydroxylation activity of Jmjd6 is required for its homooligomerization. J Cell Biochem 113(5):1663-1670

12. Bicker KL, Thompson PR (2013) The protein arginine deiminases (PADs): structure, function, inhibition, and disease. Biopolymers 99(2):155-163

13. Grant S, Easley C, Kirkpatrick P (2007) Vorinostat. Nat Rev Drug Discov 6(1):21-22

14. Bradbury RH (2007) Cancer. Topics in medicinal chemistry1st edn. Springer, Heidelberg, Berlin, p 452. XIV

15. Tamkun JW et al (1992) Brahma: a regulator of Drosophila homeotic genes structurally related to the yeast transcriptional activator SNF2SWI2. Cell 68(3):561-572

16. Filippakopoulos P, Knapp S (2014) Targeting bromodomains: epigenetic readers of lysine acetylation. Nat Rev Drug Discov 13(5):337-356

17. Yang X-J et al (1996) A p300/CBP-associated factor that competes with the adenoviral oncoprotein E1A. Nature 382(6589):319-324

18. Dhalluin C et al (1999) Structure and ligand of a histone acetyltransferase bromodomain. Nature 399(6735):491-496

19. Gregory GD et al (2007) Mammalian ASH1L is a histone methyltransferase that occupies the transcribed region of active genes. Mol Cell Biol 27(24):8466-8479

20. Malik S, Bhaumik SR (2010) Mixed lineage leukemia: histone H3 lysine 4 methyltransferases from yeast to human. FEBS J 277(8):1805-1821

21. Rahman S et al (2011) The Brd4 extraterminal domain confers transcription activation independent of pTEFb by recruiting multiple proteins, including NSD3. Mol Cell Biol 31 (13):2641-2652

22. Trotter KW, Archer TK (2008) The BRG1 transcriptional coregulator. Nucl Recept Signal 6: e004

23. Barbieri I, Cannizzaro E, Dawson MA (2013) Bromodomains as therapeutic targets in cancer. Brief Funct Genomics 12(3):219-230

24. Muller S, Filippakopoulos P, Knapp S (2011) Bromodomains as therapeutic targets. Expert Rev Mol Med 13

25. Delvecchio M et al (2013) Structure of the p300 catalytic core and implications for chromatin targeting and HAT regulation. Nat Struct Mol Biol 20(9):1040-1046

26. Kasper LH et al (1999) CREB binding protein interacts with nucleoporin-specific FG repeats that activate transcription and mediate NUP98-HOXA9 oncogenicity. Mol Cell Biol 19 (1):764-776

27. Deguchi K et al (2003) MOZ-TIF2-induced acute myeloid leukemia requires the MOZ nucleosome binding motif and TIF2-mediated recruitment of CBP. Cancer Cell 3(3):259-271

28. Picaud S et al (2015) Generation of a selective small molecule inhibitor of the CBP/p300 bromodomain for leukemia therapy. Cancer Res 75(23):5106-5119

29. Hay DA et al (2014) Discovery and optimization of small-molecule ligands for the CBP/p300 bromodomains. J Am Chem Soc 136(26):9308-9319 
30. Ciró M et al (2009) ATAD2 is a novel cofactor for MYC, overexpressed and amplified in aggressive tumors. Cancer Res 69(21):8491-8498

31. Revenko AS et al (2010) Chromatin loading of E2F-MLL complex by cancer-associated coregulator ANCCA via reading a specific histone mark. Mol Cell Biol 30(22):5260-5272

32. Leachman NT et al (2010) ATAD2B is a phylogenetically conserved nuclear protein expressed during neuronal differentiation and tumorigenesis. Dev Growth Differ 52 (9):747-755

33. Tsai W-W et al (2010) TRIM24 links a noncanonical histone signature to breast cancer. Nature 468(7326):927-932

34. Cui $\mathrm{Z}$ et al (2013) TRIM24 overexpression is common in locally advanced head and neck squamous cell carcinoma and correlates with aggressive malignant phenotypes. PLoS One 8 (5): 63887

35. Li H et al (2012) Overexpression of TRIM24 correlates with tumor progression in non-small cell lung cancer. PLoS One 7(5):e37657

36. Liu X et al (2014) Overexpression of TRIM24 is associated with the onset and progress of human hepatocellular carcinoma. PLoS One 9(1):e85462

37. Miao Z-F et al (2015) TRIM24 is upregulated in human gastric cancer and promotes gastric cancer cell growth and chemoresistance. Virchows Arch 466(5):525-532

38. Zhang LH et al (2015) TRIM24 promotes glioma progression and enhances chemoresistance through activation of the PI3K/Akt signaling pathway. Oncogene 34(5):600-610

39. Wang J et al (2014) Knockdown of tripartite motif containing 24 by lentivirus suppresses cell growth and induces apoptosis in human colorectal cancer cells. Oncol Res 22(1):39-45

40. Mujtaba S, Zeng L, Zhou MM (2007) Structure and acetyl-lysine recognition of the bromodomain. Oncogene 26(37):5521-5527

41. Vidler LR et al (2012) Druggability analysis and structural classification of bromodomain acetyl-lysine binding sites. J Med Chem 55(17):7346-7359

42. Wyce A et al (2013) Inhibition of BET bromodomain proteins as a therapeutic approach in prostate cancer. Oncotarget 4(12):2419-2429

43. Fu L-1 et al (2015) Inhibition of BET bromodomains as a therapeutic strategy for cancer drug discovery. Oncotarget 6(8):5501-5516

44. Wu S, Chiang C (2007) The double bromodomain-containing chromatin adaptor Brd4 and transcriptional regulation. J Biol Chem 282:13141-13145

45. Vollmuth F, Blankenfeldt W, Geyer M (2009) Structures of the dual bromodomains of the PTEFb-activating protein Brd4 at atomic resolution. J Biol Chem 284(52):36547-36556

46. Gamsjaeger R et al (2011) Structural basis and specificity of acetylated transcription factor GATA1 recognition by BET family bromodomain protein Brd3. Mol Cell Biol 31 (13):2632-2640

47. Sengupta $S$ et al (2015) Inhibition of BET proteins impairs estrogen-mediated growth and transcription in breast cancers by pausing RNA polymerase advancement. Breast Cancer Res Treat 150(2):265-278

48. Shang E et al (2007) The first bromodomain of Brdt, a testis-specific member of the BET sub-family of double-bromodomain-containing proteins, is essential for male germ cell differentiation. Development 134(19):3507-3515

49. Fromont $\mathrm{G}$ et al (2013) 8q24 amplification is associated with Myc expression and prostate cancer progression and is an independent predictor of recurrence after radical prostatectomy. Hum Pathol 44(8):1617-1623

50. French CA (2010) NUT midline carcinoma. Cancer Genet Cytogenet 203(1):16-20

51. Mele DA et al (2013) BET bromodomain inhibition suppresses T(H)17-mediated pathology. J Exp Med 210(11):2181-2190

52. Khan YM et al (2014) Brd4 is essential for IL-1 $\beta$-induced inflammation in human airway epithelial cells. PLoS One 9(4):e95051

53. Miyoshi S et al (2009) Antitumor agent. Patent WO2009084693 
54. Filippakopoulos P et al (2010) Selective inhibition of BET bromodomains. Nature 468 (7327):1067-1073

55. Shao Q et al (2014) BET protein inhibitor JQ1 attenuates Myc-amplified MCC tumor growth in vivo. Cancer Res 74(23):7090-7102

56. Xu C et al (2015) NSCLC driven by DDR2 mutation is sensitive to dasatinib and JQ1 combination therapy. Mol Cancer Ther 14(10):2382-2389

57. Trabucco SE et al (2015) Inhibition of bromodomain proteins for the treatment of human diffuse large B-cell lymphoma. Clin Cancer Res 21(1):113-122

58. Asangani IA et al (2014) Therapeutic targeting of BET bromodomain proteins in castrationresistant prostate cancer. Nature 510(7504):278-282

59. Picaud S et al (2013) PFI-1 - a highly selective protein interaction inhibitor targeting BET bromodomains. Cancer Res 73(11):3336-3346

60. Fish PV et al (2012) Identification of a chemical probe for bromo and extra C-terminal bromodomain inhibition through optimization of a fragment-derived hit. J Med Chem 55 (22):9831-9837

61. Yang D et al (2010) Therapeutic potential of a synthetic lethal interaction between the MYC proto-oncogene and inhibition of aurora-B kinase. Proc Natl Acad Sci U S A 107 (31):13836-13841

62. Dawson MA et al (2011) Inhibition of BET recruitment to chromatin as an effective treatment for MLL-fusion leukaemia. Nature 478(7370):529-533

63. Seal J et al (2012) Identification of a novel series of BET family bromodomain inhibitors: binding mode and profile of I-BET151 (GSK1210151A). Bioorg Med Chem Lett 22 (8):2968-2972

64. Mirguet $\mathrm{O}$ et al (2012) From ApoA1 upregulation to BET family bromodomain inhibition: discovery of I-BET151. Bioorg Med Chem Lett 22(8):2963-2967

65. Nicodeme E et al (2010) Suppression of inflammation by a synthetic histone mimic. Nature 468(7327):1119-1123

66. Mirguet O et al (2013) Discovery of epigenetic regulator I-BET762: lead optimization to afford a clinical candidate inhibitor of the BET bromodomains. J Med Chem 56 (19):7501-7515

67. French CA (2012) Pathogenesis of NUT midline carcinoma. Annu Rev Pathol 7(1):247-265

68. A study to investigate the safety, pharmacokinetics, pharmacodynamics, and clinical activity of GSK525762 in subjects with NUT midline carcinoma (NMC) and other cancers. https:// clinicaltrials.gov/ct2/show/NCT01587703?term $=$ GSK525762\&rank $=2$

69. A dose escalation study to investigate the safety, pharmacokinetics (PK), pharmacodynamics (PD) and clinical activity of GSK525762 in subjects with relapsed, refractory hematologic malignancies. https://clinicaltrials.gov/ct2/show/NCT01943851?term=GSK525762\& rank $=1$

70. Dose escalation study of GSK2820151 in subjects with advanced or recurrent solid tumors. https://clinicaltrials.gov/ct2/show/NCT02630251

71. Boi M et al (2015) The BET bromodomain inhibitor OTX015 affects pathogenetic pathways in preclinical B-cell tumor models and synergizes with targeted drugs. Clin Cancer Res 21 (7): $1628-1638$

72. Coudé M-M et al (2015) BET inhibitor OTX015 targets BRD2 and BRD4 and decreases c-MYC in acute leukemia cells. Oncotarget 6(19):17698-17712

73. Gaudio E et al (2015) The BET bromodomain inhibitor OTX015 (MK-8628) in mantle cell lymphoma (MCL): in vivo activity and identification of novel combinations to overcome adaptive resistance. In: Molecular targets and cancer therapeutics. AACR-NCI-EORTC, Boston, MA

74. Henssen AG et al (2016) Targeting MYCN-driven transcription by BET-bromodomain inhibition. Clin Cancer Res 22(10):2470-2481 
75. Odore E et al (2015) Phase I population pharmacokinetic assessment of the oral bromodomain inhibitor OTX015 in patients with haematologic malignancies. Clin Pharmacokinet 1-9

76. Sims R (2015) Discovery of CPI-0610: a novel BET protein bromodomain inhibitor for hematologic malignancies in annual meeting of the American Association for Cancer Research. AACR, Philadelphia, PA

77. Abramson JS et al (2015) BET inhibitor CPI-0610 is well tolerated and induces responses in diffuse large B-cell lymphoma and follicular lymphoma: preliminary analysis of an ongoing phase 1 study. In: 57th annual meeting \& exposition of the American Hematological Society. ASH, Orlando, $\mathrm{FL}$

78. Theodoulou NH et al (2016) Discovery of I-BRD9, a selective cell active chemical probe for bromodomain containing protein 9 inhibition. J Med Chem 59(4):1425-1439

79. Jones MH et al (2000) A novel family of bromodomain genes. Genomics 63(1):40-45

80. Strohner R et al (2001) NoRC - a novel member of mammalian ISWI-containing chromatin remodeling machines. EMBO J 20(17):4892-4900

81. Arking DE et al (2011) Identification of a sudden cardiac death susceptibility locus at 2q24.2 through genome-wide association in European ancestry individuals. PLoS Genetics 7(6): e1002158

82. Ferguson FM et al (2013) Targeting low-druggability bromodomains: fragment based screening and inhibitor design against the BAZ2B bromodomain. J Med Chem 56(24):10183-10187

83. Drouin L et al (2015) Structure enabled design of BAZ2-ICR, a chemical probe targeting the bromodomains of BAZ2A and BAZ2B. J Med Chem 58(5):2553-2559

84. Chen $P$ et al (2016) Discovery and characterization of GSK2801, a selective chemical probe for the bromodomains BAZ2A and BAZ2B. J Med Chem 59(4):1410-1424

85. Bennett $\mathrm{J}$ et al (2016) Discovery of a chemical tool inhibitor targeting the bromodomains of TRIM24 and BRPF. J Med Chem 59(4):1642-1647

86. Palmer WS et al (2016) Structure-guided design of IACS-9571, a selective high-affinity dual TRIM24-BRPF1 bromodomain inhibitor. J Med Chem 59(4):1440-1454

87. Oike $\mathrm{T}$ et al (2013) A synthetic lethality-based strategy to treat cancers harboring a genetic deficiency in the chromatin remodeling factor BRG1. Cancer Res 73(17):5508-5518

88. Hoffman GR et al (2014) Functional epigenetics approach identifies BRM/SMARCA2 as a critical synthetic lethal target in BRG1-deficient cancers. Proc Natl Acad Sci U S A 111 (8):3128-3133

89. Wilson BG et al (2014) Residual complexes containing SMARCA2 (BRM) underlie the oncogenic drive of SMARCA4 (BRG1) mutation. Mol Cell Biol 34(6):1136-1144

90. Vangamudi B et al (2015) The SMARCA2/4 ATPase domain surpasses the bromodomain as a drug target in SWI/SNF mutant cancers: insights from cDNA rescue and PFI-3 inhibitor studies. Cancer Res 75(18):3865-3878

91. Cho EC et al (2012) Arginine methylation controls growth regulation by E2F-1. EMBO J 31:1785-1797

92. Chung J et al (2013) Protein arginine methyltransferase 5 (PRMT5) inhibition induces lymphoma cell death through reactivation of the retinoblastoma tumor suppressor pathway and polycomb repressor complex 2 (PRC2) silencing. J Biol Chem 288(49):35534-35547

93. Pal S et al (2007) Low levels of miR-92b/96 induce PRMT5 translation and H3R8/H4R3 methylation in mantle cell lymphoma. 26 3558-3569

94. Pal S et al (2004) Human SWI/SNF-associated PRMT5 methylates histone $\mathrm{H} 3$ arginine 8 and negatively regulates expression of ST7 and NM23 tumor suppressor genes. Mol Cell Biol 24 (21):9630-9645

95. Powers MA et al (2011) Protein arginine methyltransferase 5 accelerates tumor growth by arginine methylation of the tumor suppressor programmed cell death 4. Cancer Res 71 (16):5579-5587 
96. Wang L, Pal S, Sif S (2008) Protein arginine methyltransferase 5 suppresses the transcription of the RB family of tumor suppressors in leukemia and lymphoma cells. Mol Cell Biol 28 (20):6262-6277

97. Wei T-YW et al (2012) Protein arginine methyltransferase 5 is a potential oncoprotein that upregulates G1 cyclins/cyclin-dependent kinases and the phosphoinositide 3-kinase/AKT signaling cascade. Cancer Sci 103(9):1640-1650

98. Karkhanis V et al (2011) Versatility of PRMT5-induced methylation in growth control and development. Trends Biochem Sci 36(12):633-641

99. Koh CM et al (2015) MYC regulates the core pre-mRNA splicing machinery as an essential step in lymphomagenesis. Nature 523(7558):96-100

100. Antonysamy S et al (2012) Crystal structure of the human PRMT5:MEP50 complex. Proc Natl Acad Sci 109(44):17960-17965

101. Smil D et al (2015) Discovery of a dual PRMT5-PRMT7 inhibitor. ACS Med Chem Lett 6 (4):408-412

102. Alinari L et al (2015) Selective inhibition of protein arginine methyltransferase 5 blocks initiation and maintenance of B-cell transformation. Blood 125(16):2530-2543

103. Chan-Penebre E et al (2015) A selective inhibitor of PRMT5 with in vivo and in vitro potency in MCL models. Nat Chem Biol 11(6):432-437

104. Duncan KW et al (2015) Structure and property guided design in the identification of PRMT5 tool compound EPZ015666. ACS Med Chem Lett 7(2):162-166

105. Dose escalation study of GSK3326595 in subjects with solid tumors and non-Hodgkin's lymphoma. $\quad$ https://clinicaltrials.gov/ct2/show/NCT02783300?term =NCT02783300\& rank $=1$

106. El-Andaloussi N et al (2006) Arginine methylation regulates DNA polymerase beta. Mol Cell 22(1):51

107. Kleinschmidt MA et al (2012) Cell cycle regulation by the PRMT6 arginine methyltransferase through repression of cyclin-dependent kinase inhibitors. PLoS One 7(8): e41446

108. Yoshimatsu M et al (2011) Dysregulation of PRMT1 and PRMT6, type I arginine methyltransferases, is involved in various types of human cancers. Int J Cancer 128(3):562

109. Vieira FQ et al (2014) Deregulated expression of selected histone methylases and demethylases in prostate carcinoma. Endocr Relat Cancer 21(1):51

110. Limm K et al (2013) Deregulation of protein methylation in melanoma. Eur J Cancer 49 (6): 1305

111. Mitchell LH et al (2015) Aryl pyrazoles as potent inhibitors of arginine methyltransferases: identification of the first PRMT6 tool compound. ACS Med Chem Lett 6(6):655-659

112. Kaniskan HÜ et al (2015) A potent, selective and cell-active allosteric inhibitor of protein arginine methyltransferase 3 (PRMT3). Angew Chem Int Edit 54(17):5166-5170

113. Liu F et al (2013) Exploiting an allosteric binding site of PRMT3 yields potent and selective inhibitors. J Med Chem 56(5):2110-2124

114. Feng Q et al (2002) Methylation of H3-lysine 79 is mediated by a new family of HMTases without a SET domain. Curr Biol 12(12):1052-1058

115. Lacoste $\mathrm{N}$ et al (2002) Disruptor of telomeric silencing-1 is a chromatin-specific histone $\mathrm{H} 3$ methyltransferase. J Biol Chem 277(34):30421-30424

116. $\mathrm{Ng} \mathrm{HH}$ et al (2002) Lysine methylation within the globular domain of histone H3 by Dot1 is important for telomeric silencing and Sir protein association. Genes Dev 16(12):1518-1527

117. van Leeuwen F, Gafken PR, Gottschling DE (2002) Dot1p modulates silencing in yeast by methylation of the nucleosome core. Cell 109(6):745-756

118. Min J et al (2003) Structure of the catalytic domain of human DOT1L, a non-SET domain nucleosomal histone methyltransferase. Cell 112(5):711-723

119. Frederiks F et al (2008) Nonprocessive methylation by Dot1 leads to functional redundancy of histone H3K79 methylation states. Nat Struct Mol Biol 15(6):550-557 
120. Nguyen AT, Zhang Y (2011) The diverse functions of Dot1 and H3K79 methylation. Genes Dev 25(13):1345-1358

121. Hess JL (2004) MLL: a histone methyltransferase disrupted in leukemia. Trends Mol Med 10 (10):500-507

122. Krivtsov AV, Armstrong SA (2007) MLL translocations, histone modifications and leukaemia stem-cell development. Nat Rev Cancer 7(11):823-833

123. Milne TA et al (2002) MLL targets SET domain methyltransferase activity to Hox gene promoters. Mol Cell 10(5):1107-1117

124. Nakamura T et al (2002) ALL-1 is a histone methyltransferase that assembles a supercomplex of proteins involved in transcriptional regulation. Mol Cell 10(5):1119-1128

125. Guenther MG et al (2008) Aberrant chromatin at genes encoding stem cell regulators in human mixed-lineage leukemia. Genes Dev 22(24):3403-3408

126. Krivtsov AV et al (2008) H3K79 methylation profiles define murine and human MLL-AF4 leukemias. Cancer Cell 14(5):355-368

127. Richon VM et al (2011) Chemogenetic analysis of human protein methyltransferases. Chem Biol Drug Des 78(2):199-210

128. Daigle SR et al (2011) Selective killing of mixed lineage leukemia cells by a potent smallmolecule DOT1L inhibitor. Cancer Cell 20(1):53-65

129. Basavapathruni A et al (2012) Conformational adaptation drives potent, selective and durable inhibition of the human protein methyltransferase DOT1L. Chem Biol Drug Des 80 (6):971-980

130. Armstrong SA et al (2003) Inhibition of FLT3 in MLL: validation of a therapeutic target identified by gene expression based classification. Cancer Cell 3(2):173-183

131. Chesworth R et al (2011) 7-Deazapurine modulators of histone methyltransferase, and methods of use thereof in. Patent WO2012075500

132. Yao Y et al (2011) Selective inhibitors of histone methyltransferase DOT1L: design, synthesis and crystallographic studies. J Am Chem Soc 133(42):16746-16749

133. Song $Y$ et al (2014) Selective inhibitors of histone methyltransferase dot1l. Patent US20140100184

134. Lee JM, Choi M (2014) Compounds and compositions for modulating histone methyltransferase activity. Patent WO2014035140

135. Daigle SR et al (2013) Potent inhibition of DOT1L as treatment of MLL-fusion leukemia. Blood 122(6): 1017-1025

136. Epizyme I. A phase 1 dose escalation and expanded cohort study of EPZ-5676 in the treatment of pediatric patients with relapsed/refractory leukemias bearing a rearrangement of the MLL gene. https://clinicaltrials.gov/ct2/show/NCT02141828?term=5676\&rank=1

137. Waters NJ et al (2015) Metabolism and disposition of the DOT1L inhibitor, pinometostat (EPZ-5676), in rat, dog and human. Cancer Chemother Pharmacol 77(1):43-62

138. Stein EM et al (2015) A phase 1 study of the DOT1L inhibitor, pinometostat (EPZ-5676), in adults with relapsed or refractory leukemia: safety, clinical activity, exposure and target inhibition, in 57th annual meeting \& exposition. American Association of Hematologists, Orlando, FL

139. Raimondi A et al (2015) Abstract B82: pinometostat (EPZ-5676) enhances the antiproliferative activity of MAP kinase pathway inhibitors in MLL-rearranged leukemia cell lines. Mol Cancer Therapeut 14(12 Suppl 2):B82

140. Finley A, Copeland RA (2014) Small molecule control of chromatin remodeling. Chem Biol 21(9):1196-1210

141. Morin RD et al (2010) Somatic mutation of EZH2 (Y641) in follicular and diffuse large B-cell lymphomas of germinal center origin. Nat Genet 42(2):181-185

142. Wilson BG et al (2010) Epigenetic antagonism between polycomb and SWI/SNF complexes during oncogenic transformation. Cancer Cell 18(4):316-328

143. Gibson WT et al (2012) Mutations in EZH2 cause weaver syndrome. Am J Hum Genet 90 (1):110-118 
144. LaFave LM et al (2015) Loss of BAP1 function leads to EZH2-dependent transformation. Nat Med 21(11):1344-1349

145. Ntziachristos P et al (2012) Genetic inactivation of the PRC2 complex in T-cell acute lymphoblastic leukemia. Nat Med 18(2):298-301

146. Simon C et al (2012) A key role for EZH2 and associated genes in mouse and human adult T-cell acute leukemia. Genes Dev 26(7):651-656

147. Shih AH et al (2012) The role of mutations in epigenetic regulators in myeloid malignancies. Nat Rev Cancer 12(9):599-612

148. Brown R et al (2014) Poised epigenetic states and acquired drug resistance in cancer. Nat Rev Cancer 14(11):747-753

149. Kim E et al (2013) Phosphorylation of EZH2 activates STAT3 signaling via STAT3 methylation and promotes tumorigenicity of glioblastoma stem-like cells. Cancer Cell 23 (6):839-852

150. de Vries NA et al (2015) Prolonged Ezh2 depletion in glioblastoma causes a robust switch in cell fate resulting in tumor progression. Cell Rep 10(3):383-397

151. Basso K, Dalla-Favera R (2015) Germinal centres and B cell lymphomagenesis. Nat Rev Immunol 15(3):172-184

152. Johnston D et al (2015) EZH2 plays a critical role in B cell maturation and in non-Hodgkin's lymphoma: interplay between EZH2 function and B-cell activation. In: 57th annual meeting and exposition. American Society of Hematologists, Orlando, FL

153. Swalm BM et al (2014) Reaction coupling between wild-type and disease-associated mutant EZH2. ACS Chem Biol 9(11):2459-2464

154. Shain AH et al (2012) Convergent structural alterations define SWItch/sucrose nonfermentable (SWI/SNF) chromatin remodeler as a central tumor suppressive complex in pancreatic cancer. Proc Natl Acad Sci U S A 109(5):E252-E259

155. Shain AH, Pollack JR (2013) The spectrum of SWI/SNF mutations, ubiquitous in human cancers. PLoS One 8(1):e55119

156. Knutson SK et al (2013) Durable tumor regression in genetically altered malignant rhabdoid tumors by inhibition of methyltransferase EZH2. Proc Natl Acad Sci U S A 110 (19):7922-7927

157. Errico A (2014) Genetics: SMARCA4 mutated in SCCOHT. Nat Rev Clin Oncol 11 (6):302-302

158. Knutson SK et al (2014) Synergistic anti-tumor activity of EZH2 inhibitors and glucocorticoid receptor agonists in models of germinal center non-Hodgkin lymphomas. PLoS One 9 (12):e111840

159. Cao R et al (2002) Role of histone H3 lysine 27 methylation in polycomb-group silencing. Science 298(5595):1039-1043

160. Jiao L, Liu X (2015) Structural basis of histone H3K27 trimethylation by an active polycomb repressive complex 2. Science 350(6258)

161. Knutson SK et al (2012) A selective inhibitor of EZH2 blocks H3K27 methylation and kills mutant lymphoma cells. Nat Chem Biol 8(11):890-896

162. McCabe MT et al (2012) EZH2 inhibition as a therapeutic strategy for lymphoma with EZH2activating mutations. Nature 492(7427):108-112

163. Verma SK et al (2012) Identification of potent, selective, cell-active inhibitors of the histone lysine methyltransferase EZH2. ACS Med Chem Lett 3(12):1091-1096

164. Qi W et al (2012) Selective inhibition of Ezh2 by a small molecule inhibitor blocks tumor cells proliferation. Proc Natl Acad Sci U S A 109(52):21360-21365

165. Konze KD et al (2013) An orally bioavailable chemical probe of the lysine methyltransferases EZH2 and EZH1. ACS Chem Biol 8(6):1324-1334

166. Kuntz KW et al (2016) The importance of being me: magic methyls, methyltransferase inhibitors, and the discovery of tazemetostat. J Med Chem 59(4):1556-1564

167. Campbell JE et al (2015) EPZ011989, a potent, orally-available EZH2 inhibitor with robust in vivo activity. ACS Med Chem Lett 6(5):491-495 
168. Nasveschuk CG et al (2014) Discovery and optimization of tetramethylpiperidinyl benzamides as inhibitors of EZH2. ACS Med Chem Lett 5(4):378-383

169. Kung P-P et al (2015) SAH derived potent and selective EZH2 inhibitors. Bioorg Med Chem Lett 25(7):1532-1537

170. Bradley WD et al (2014) EZH2 inhibitor efficacy in non-Hodgkin's lymphoma does not require suppression of H3K27 monomethylation. Chem Biol 21(11):1463-1475

171. Vaswani RG et al (2016) Identification of (R)-N-((4-methoxy-6-methyl-2-oxo-1,2dihydropyridin-3-yl)methyl)-2-methyl-1-(1-(1-(2,2,2-trifluoroethyl)piperidin-4-yl)ethyl)-

1H-indole-3-carboxamide (CPI-1205), a potent and selective inhibitor of histone methyltransferase EZH2, suitable for phase I clinical trials for B-cell lymphomas. J Med Chem 59(21):9928-9941

172. A study evaluating CPI-1205 in patients with B-cell lymphomas. https://clinicaltrials.gov/ ct2/show/NCT02395601

173. Baker T et al (2015) Acquisition of a single EZH2 D1 domain mutation confers acquired resistance to EZH2-targeted inhibitors. Oncotarget 6(32)

174. Kim W et al (2013) Targeted disruption of the EZH2/EED complex inhibits EZH2-dependent cancer. Nat Chem Biol 9(10):643-650

175. Shankar SR et al (2013) G9a, a multipotent regulator of gene expression. Epigenetics 8 (1):16-22

176. Chang $\mathrm{Y}$ et al (2010) Adding a lysine mimic in the design of potent inhibitors of histone lysine methyltransferases. J Mol Biol 400(1):1-7

177. Vedadi M et al (2011) A chemical probe selectively inhibits G9a and GLP methyltransferase activity in cells. Nat Chem Biol 7(8):566-574

178. Sweis RF et al (2014) Discovery and development of potent and selective inhibitors of histone methyltransferase G9a. ACS Med Chem Lett 5(2):205-209

179. Pappano WN et al (2015) The histone methyltransferase inhibitor A-366 uncovers a role for G9a/GLP in the epigenetics of leukemia. PLoS One 10(7):e0131716

180. Liu S et al (2015) G9a is essential for EMT-mediated metastasis and maintenance of cancer stem cell-like characters in head and neck squamous cell carcinoma. Oncotarget 6 (9):6887-6901

181. Zhong X et al (2015) Overexpression of G9a and MCM7 in oesophageal squamous cell carcinoma is associated with poor prognosis. Histopathology 66(2):192-200

182. Tao $\mathrm{H}$ et al (2014) Histone methyltransferase G9a and H3K9 dimethylation inhibit the selfrenewal of glioma cancer stem cells. Mol Cell Biochem 394(1):23-30

183. Chaturvedi C-P et al (2009) Dual role for the methyltransferase G9a in the maintenance of $\beta$-globin gene transcription in adult erythroid cells. Proc Natl Acad Sci U S A 106 (43): $18303-18308$

184. Renneville A et al (2015) EHMT1 and EHMT2 inhibition induces fetal hemoglobin expression. Blood 126(16):1930-1939

185. Krivega I et al (2015) Inhibition of G9a methyltransferase stimulates fetal hemoglobin production by facilitating LCR/ $\gamma$-globin looping. Blood 126(5):665-672

186. Zhang $X$ et al (2013) Regulation of estrogen receptor $\alpha$ by histone methyltransferase SMYD2-mediated protein methylation. Proc Natl Acad Sci U S A 110(43):17284-17289

187. Piao L et al (2014) The histone methyltransferase SMYD2 methylates PARP1 and promotes poly(ADP-ribosyl)ation activity in cancer cells. Neoplasia 16(3):257-264.e2

188. Brown MA et al (2006) Identification and characterization of Smyd2: a split SET/MYND domain-containing histone $\mathrm{H} 3$ lysine 36-specific methyltransferase that interacts with the Sin3 histone deacetylase complex. Mol Cancer 5:26-26

189. Huang J et al (2006) Repression of p53 activity by Smyd2-mediated methylation. Nature 444 (7119):629-632

190. Komatsu S et al (2009) Overexpression of SMYD2 relates to tumor cell proliferation and malignant outcome of esophageal squamous cell carcinoma. Carcinogenesis 30(7):1139-1146 
191. Komatsu S et al (2015) Overexpression of SMYD2 contributes to malignant outcome in gastric cancer. Br J Cancer 112(2):357-364

192. Sakamoto LHT et al (2014) SMYD2 is highly expressed in pediatric acute lymphoblastic leukemia and constitutes a bad prognostic factor. Leuk Res 38(4):496-502

193. Ferguson AD et al (2011) Structural basis of substrate methylation and inhibition of SMYD2. Structure 19(9):1262-1273

194. Sweis RF et al (2015) Discovery of A-893, a new cell-active benzoxazinone inhibitor of lysine methyltransferase SMYD2. ACS Med Chem Lett 6(6):695-700

195. Nguyen H et al (2015) LLY-507, a cell-active, potent, and selective inhibitor of protein-lysine methyltransferase SMYD2. J Biol Chem 290(22):13641-13653

196. Stresemann C et al (2015) Abstract 2829: discovery and in vitro and in vivo characterization of aminopyrazoline-based SMYD2 inhibitors. Cancer Res 75(Suppl 15):2829

197. Hamamoto R et al (2006) Enhanced SMYD3 expression is essential for the growth of breast cancer cells. Cancer Sci 97(2):113-118

198. Vieira FQ et al (2015) SMYD3 contributes to a more aggressive phenotype of prostate cancer and targets Cyclin D2 through H4K20me3. Oncotarget 6(15):13644-13657

199. Mazur PK et al (2014) SMYD3 links lysine methylation of MAP3K2 to Ras-driven cancer. Nature 510(7504):283-287

200. Liu Y et al (2015) Overexpression of SMYD3 and matrix metalloproteinase-9 are associated with poor prognosis of patients with gastric cancer. Tumor Biol 36(6):4377-4386

201. Peserico A et al (2015) A SMYD3 small-molecule inhibitor impairing cancer cell growth. J Cell Physiol 230(10):2447-2460

202. Keating ST et al (2014) Deep sequencing reveals novel Set7 networks. Cell Mol Life Sci 71 (22):4471-4486

203. Barsyte-Lovejoy D et al (2014) (R)-PFI-2 is a potent and selective inhibitor of SETD7 methyltransferase activity in cells. Proc Natl Acad Sci U S A 111(35):12853-12858

204. He S et al (2015) Lysine methyltransferase SETD7 (SET7/9) regulates ROS signaling through mitochondria and NFE2L2/ARE pathway. Sci Rep 5:14368

205. Bannister AJ, Schneider R, Kouzarides T (2002) Histone methylation: dynamic or static? Cell 109(7):801-806

206. Cloos PAC et al (2008) Erasing the methyl mark: histone demethylases at the center of cellular differentiation and disease. Genes Dev 22(9):1115-1140

207. Culhane JC, Cole PA (2007) LSD1 and the chemistry of histone demethylation. Curr Opin Chem Biol 11(5):561-568

208. Mohammad HP et al (2015) A DNA hypomethylation signature predicts antitumor activity of LSD1 inhibitors in SCLC. Cancer Cell 28(1):57-69

209. Wang Y et al (2009) LSD1 is a subunit of the NuRD complex and targets the metastasis programs in breast cancer. Cell 138(4):660-672

210. Kahl P et al (2006) Androgen receptor coactivators lysine-specific histone demethylase 1 and four and a half LIM domain protein 2 predict risk of prostate cancer recurrence. Cancer Res 66(23):11341-11347

211. Schenk T et al (2012) Inhibition of the LSD1 (KDM1A) demethylase reactivates the all-transretinoic acid differentiation pathway in acute myeloid leukemia. Nat Med 18(4):605-611

212. Harris WJ et al (2012) The histone demethylase KDM1A sustains the oncogenic potential of MLL-AF9 leukemia stem cells. Cancer Cell 21(4):473-487

213. Wada $\mathrm{T}$ et al (2015) Overexpression of the shortest isoform of histone demethylase LSD1 primes hematopoietic stem cells for malignant transformation. Blood 125(24):3731-3746

214. Lee MG et al (2005) An essential role for CoREST in nucleosomal histone 3 lysine 4 demethylation. Nature 437(7057):432-435

215. Metzger E et al (2005) LSD1 demethylates repressive histone marks to promote androgenreceptor-dependent transcription. Nature 437(7057):436-439 
216. Biswas D et al (2011) Function of leukemogenic mixed lineage leukemia 1 (MLL) fusion proteins through distinct partner protein complexes. Proc Natl Acad Sci U S A 108 (38): $15751-15756$

217. Chen Y et al (2006) Crystal structure of human histone lysine-specific demethylase 1 (LSD1). Proc Natl Acad Sci U S A 103(38):13956-13961

218. Mimasu S et al (2008) Crystal structure of histone demethylase LSD1 and tranylcypromine at $2.25 \AA$ A. Biochem Biophys Res Commun 366(1):15-22

219. Singh J et al (2011) The resurgence of covalent drugs. Nat Rev Drug Discov 10(4):307-317

220. Fiedorowicz JG, Swartz KL (2004) The role of monoamine oxidase inhibitors in current psychiatric practice. J Psychiatr Pract 10(4):239-248

221. Schmidt DMZ, McCafferty DG (2007) Trans-2-phenylcyclopropylamine is a mechanismbased inactivator of the histone demethylase LSD1. Biochemistry 46(14):4408-4416

222. Yang M et al (2007) Structural basis for the inhibition of the LSD1 histone demethylase by the antidepressant trans-2-phenylcyclopropylamine. Biochemistry 46(27):8058-8065

223. Yi CZ et al (2016) Irreversible LSD1 inhibitors: application of tranylcypromine and its derivatives in cancer treatment. Curr Top Med Chem 16:1-1

224. Schmitt ML et al (2013) Non-peptidic propargylamines as inhibitors of lysine specific demethylase 1 (LSD1) with cellular activity. J Med Chem 56(18):7334-7342

225. Prusevich $P$ et al (2014) A selective phenelzine analogue inhibitor of histone demethylase LSD1. ACS Chem Biol 9(6):1284-1293

226. Mould DP et al (2015) Reversible inhibitors of LSD1 as therapeutic agents in acute myeloid leukemia: clinical significance and progress to date. Med Res Rev 35(3):586-618

227. Huang $Y$ et al (2009) Novel oligoamine analogues inhibit lysine-specific demethylase 1 (LSD1) and induce re-expression of epigenetically silenced genes. Clin Cancer Res 15 (23):7217-7228

228. Hazeldine $\mathrm{S}$ et al (2012) Low molecular weight amidoximes that act as potent inhibitors of lysine-specific demethylase 1. J Med Chem 55(17):7378-7391

229. Sorna V et al (2013) High-throughput virtual screening identifies novel $\mathrm{N}^{\prime}$-(1-phenylethylidene)-benzohydrazides as potent, specific, and reversible LSD1 inhibitors. J Med Chem 56(23):9496-9508

230. Sankar S et al (2014) Reversible LSD1 inhibition interferes with global EWS/ETS transcriptional activity and impedes Ewing sarcoma tumor growth. Clin Cancer Res 20 (17):4584-4597

231. Baell JB, Holloway GA (2010) New substructure filters for removal of pan assay interference compounds (PAINS) from screening libraries and for their exclusion in bioassays. J Med Chem 53(7):2719-2740

232. Hitchin JR et al (2013) Development and evaluation of selective, reversible LSD1 inhibitors derived from fragments. Med Chem Commun 4(11):1513-1522

233. Zheng Y-C et al (2013) Triazole-dithiocarbamate based, selective LSD1 inactivators inhibit gastric cancer cell growth, invasion and migration. J Med Chem 56(21). https://doi.org/10. $1021 / \mathrm{jm} 401002 \mathrm{r}$

234. Dhanak D (2013) Proceedings of the 104th annual meeting of the American Association for Cancer Research

235. Investigation of GSK2879552 in subjects with relapsed/refractory small cell lung carcinoma. https://clinicaltrials.gov/ct2/show/NCT02034123?term=GSK2879552\&rank=1

236. A phase I dose escalation study of GSK2879552 in subjects with acute myeloid leukemia (AML). https://clinicaltrials.gov/ct2/show/NCT02177812?term $=$ GSK2879552\&rank $=2$

237. A phase I study of human pharmacokinetics and safety of ORY-1001, and LSD1 inhibitor, in relapsed or refractory acute leukaemia (AL). https://www.clinicaltrialsregister.eu/ctr-search/ trial/2013-002447-29/ES

238. McAllister TE et al (2015) Recent progress in histone demethylase inhibitors. J Med Chem

239. Hoffmann I et al (2012) The role of histone demethylases in cancer therapy. Mol Oncol 6 (6):683-703 
240. Cheng $\mathrm{Z}$ et al (2014) A molecular threading mechanism underlies Jumonji lysine demethylase KDM2A regulation of methylated H3K36. Genes Dev 28(16):1758-1771

241. Frescas D et al (2008) KDM2A represses transcription of centromeric satellite repeats and maintains the heterochromatic state. Cell Cycle 7(22):3539-3547

242. Lu T et al (2009) Validation-based insertional mutagenesis identifies lysine demethylase FBXL11 as a negative regulator of NFкB. Proc Natl Acad Sci U S A 106(38):16339-16344

243. Rizwani W et al (2014) Mammalian lysine histone demethylase KDM2A regulates E2F1mediated gene transcription in breast cancer cells. PLoS One 9(7):e100888

244. England KS et al (2014) Optimisation of a triazolopyridine based histone demethylase inhibitor yields a potent and selective KDM2A (FBXL11) inhibitor. Med Chem Commun 5 (12):1879-1886

245. Klose RJ et al (2007) The retinoblastoma binding protein RBP2 is an H3K4 demethylase. Cell 128(5):889-900

246. Christensen J et al (2007) RBP2 belongs to a family of demethylases, specific for tri- and dimethylated lysine 4 on histone 3. Cell 128(6):1063-1076

247. Uemura M et al (2010) Jumonji domain containing $1 \mathrm{~A}$ is a novel prognostic marker for colorectal cancer: in vivo identification from hypoxic tumor cells. Clin Cancer Res 16 (18):4636-4646

248. Teng Y-C et al (2013) Histone demethylase RBP2 promotes lung tumorigenesis and cancer metastasis. Cancer Res 73(15):4711-4721

249. Lin W et al (2011) Loss of the retinoblastoma binding protein 2 (RBP2) histone demethylase suppresses tumorigenesis in mice lacking Rb1 or Men1. Proc Natl Acad Sci U S A 108 (33):13379-13386

250. Hou $\mathrm{J}$ et al (2012) Genomic amplification and a role in drug-resistance for the KDM5A histone demethylase in breast cancer. Am J Transl Res 4(3):247-256

251. de Rooij JDE et al (2013) NUP98/JARID1A is a novel recurrent abnormality in pediatric acute megakaryoblastic leukemia with a distinct HOX gene expression pattern. Leukemia 27 (12):2280-2288

252. Albrecht BK et al (2015) Therapeutic compounds and uses thereof. Patent WO2015135094

253. Vinogradova M et al (2016) An inhibitor of KDM5 demethylases reduces survival of drugtolerant cancer cells. Nat Chem Biol 12(7):531-538

254. Gehling VS et al (2016) Identification of potent, selective KDM5 inhibitors. Bioorg Med Chem Lett 26(17):4350-4354

255. Hong S et al (2007) Identification of JmjC domain-containing UTX and JMJD3 as histone H3 lysine 27 demethylases. Proc Natl Acad Sci 104(47):18439-18444

256. van Haaften G et al (2009) Somatic mutations of the histone H3K27 demethylase, UTX, in human cancer. Nat Genet 41(5):521-523

257. Lederer D et al (2012) Deletion of KDM6A, a histone demethylase interacting with MLL2, in three patients with kabuki syndrome. Am J Hum Genet 90(1):119-124

258. Walport LJ et al (2014) Human UTY(KDM6C) is a male-specific N(€)-methyl lysyl demethylase. J Biol Chem 289(26):18302-18313

259. Kruidenier L et al (2012) A selective jumonji H3K27 demethylase inhibitor modulates the proinflammatory macrophage response. Nature 488(7411):404-408

260. Heinemann B et al (2014) Inhibition of demethylases by GSK-J1/J4. Nature 514(7520):E1E2

261. Shi J et al (2015) Discovery of cancer drug targets by CRISPR-Cas9 screening of protein domains. Nat Biotechnol 33(6):661-667 